\title{
Elliptic equations with vertical asymptotes in the nonlinear term
}

Louis Dupaigne, Augusto C. Ponce and Alessio Porretta

June 22, 2005

Abstract. We study the existence of solutions of the nonlinear problem

$$
\left\{\begin{array}{rlrl}
-\Delta u+g(u) & =\mu & & \text { in } \Omega, \\
u=0 & & \text { on } \partial \Omega,
\end{array}\right.
$$

where $\mu$ is a bounded measure and $g$ is a continuous nondecreasing function such that $g(0)=0$. In this paper, we assume that the nonlinearity $g$ satisfies

$$
\lim _{t \uparrow 1} g(t)=+\infty .
$$

Problem (0.1) need not have a solution for every measure $\mu$. We prove that, given $\mu$, there exists a "closest" measure $\mu^{*}$ for which (0.1) can be solved. We also explain how assumption (0.2) makes problem (0.1) different compared to the case where $g(t)$ is defined for every $t \in \mathbb{R}$.

(Paper to appear in J. Anal. Math.)

Mathematics Subject Classification (2000). 28A78, 35B05, 35B45, 35B65, 35J60

Key words. Elliptic equations involving measures, nonlinearities with vertical asymptotes, reduced measures

\section{Introduction}

Let $\Omega \subset \mathbb{R}^{N}, N \geq 2$, be a smooth bounded domain. In this paper, we are interested in the existence of solutions of the following problem

$$
\left\{\begin{aligned}
-\Delta u+g(u)=\mu & & \text { in } \Omega, \\
u=0 & & \text { on } \partial \Omega,
\end{aligned}\right.
$$

where $\mu$ is a bounded measure in $\Omega$ and $g:(-\infty, 1) \rightarrow \mathbb{R}$ is a continuous nondecreasing function such that $g(0)=0$ and

$$
\lim _{t \uparrow 1} g(t)=+\infty
$$


By a solution $u$ of $(1.1)$ we mean that $u \in L^{1}(\Omega), u \leq 1$ a.e., $g(u) \in L^{1}(\Omega)$ and

$$
-\int_{\Omega} u \Delta \zeta+\int_{\Omega} g(u) \zeta=\int_{\Omega} \zeta d \mu \quad \forall \zeta \in C^{2}(\bar{\Omega}), \zeta=0 \text { on } \partial \Omega .
$$

In particular, $g(u) \in L^{1}(\Omega)$ implies that $u<1$ a.e.

We observe that $u$, whenever it exists, is unique (see e.g. [4]). It has been proved by Boccardo [2] (in the spirit of Brezis-Strauss [7]) that, for every $\mu \in L^{1}(\Omega)$, problem (1.1) has a solution. Moreover, Boccardo also shows that (1.1) has no solution if $\mu$ is a Dirac mass $\delta_{a}$, with $a \in \Omega$. Consequently, we say that $\mu$ is a good measure (relative to $g$ ) if (1.1) has a solution $u$. We shall denote by $\mathcal{G}(g)$ the set of good measures associated to $g$.

Our goal in this paper is to investigate under what conditions on $g$ and $\mu$ problem (1.1) admits a solution. We also point out to what extent assumption (1.2) makes this problem different compared to the case where $g$ is a continuous function defined for every $t \in \mathbb{R}$, which was recently studied by Brezis-MarcusPonce [4].

We shall assume henceforth that, in addition to (1.2), $g$ satisfies

$$
g(t)=0 \quad \forall t \leq 0
$$

In particular, this implies that nonpositive measures are good for any $g$.

We denote by $\mathcal{M}(\Omega)$ the space of bounded Radon measures in $\Omega$, equipped with its standard norm \|\|$_{\mathcal{M}}$. Given $\nu \in \mathcal{M}(\Omega)$, we say that $\nu$ is diffuse if $\nu(A)=0$ for every Borel set $A \subset \Omega$ of zero $H^{1}$-capacity (= Newtonian capacity). As we shall see, this capacity — which will be denoted throughout this paper by "cap" - plays an important role in the study of problem (1.1).

The first consequence of (1.2) is that if (1.1) has a solution, then $\mu^{+}$is diffuse (see Corollary 2 in Section 2 below). The converse is not true; more precisely,

Theorem 1 Given any $g$, there exists a diffuse measure $\mu \geq 0$ such that $\mu \notin \mathcal{G}(g)$.

However, we shall see later on that every diffuse measure is good for some $g$ (see Theorem 15).

For a fixed nonlinearity $g$, a natural question is to characterize the set of good measures associated to $g$. The next result gives a sufficient condition for a measure to be good: 
Theorem 2 Assume

$$
\limsup _{t \uparrow 1}\left\{(1-t)^{\frac{2-\beta}{\beta}} g(t)\right\}>0
$$

for some $0<\beta<2$. If $\mu^{+} \ll \mathcal{H}^{N-2+\beta}$, then $\mu \in \mathcal{G}(g)$.

Here, $\mathcal{H}^{s}$ denotes the $s$-dimensional Hausdorff measure of a set. By $\mu^{+} \ll \mathcal{H}^{s}$, we mean that $\mu^{+}(A)=0$ for every Borel set $A \subset \Omega$ such that $\mathcal{H}^{s}(A)=0$. We point out that the dimension $s=N-2+\beta$ in the statement of the theorem cannot be improved. In fact, given $\beta \in(0,2)$, let

$$
g(t)=\frac{1}{(1-t)^{\frac{2-\beta}{\beta}}}-1 \quad \forall t \in[0,1) .
$$

For any $\alpha<N-2+\beta$, one can find a compact set $K_{\alpha} \subset \Omega$, with $\mathcal{H}^{\alpha}\left(K_{\alpha}\right) \in(0, \infty)$, such that if $\theta>0$ is sufficiently large, then $\mu=\theta \mathcal{H}^{\alpha}\left\lfloor_{K_{\alpha}}\right.$ is not good for $g$. This is easy to see if $\alpha \leq N-2$ since in this case any compact set $K \subset \Omega$ such that $\mathcal{H}^{\alpha}(K)<\infty$ satisfies cap $(K)=0$ (see e.g. [8]); thus, by Corollary 2 in Section $2, \mu$ is not good. In the remaining case, namely $N-2<\alpha<N-2+\beta$, the construction of $K_{\alpha}$ is rather delicate and will be presented in Section 8 (see Theorem 18).

Even though the existence of solutions of problem (1.1) may fail for some diffuse measures (by Theorem 1 ), $L^{1}(\Omega)$ is not the largest set where (1.1) has a solution for any $g$. For instance, let $\mu \in \mathcal{M}(\Omega)$ be such that $v \leq 1$ a.e., where $v$ is the unique solution of

$$
\left\{\begin{aligned}
-\Delta v=\mu & \text { in } \Omega, \\
v=0 & \text { on } \partial \Omega .
\end{aligned}\right.
$$

Then, $\mu$ is good for every $g$ (see Proposition 7 in Section 7 ). The converse is also true if $\mu^{+}$is singular with respect to the Lebesgue measure in $\mathbb{R}^{N}$. In fact, we have the following

Theorem 3 Let $\mu \in \mathcal{M}(\Omega)$ be such that $\mu^{+}$is singular. Then, $\mu \in \mathcal{G}(g)$ for every $g$ if and only if $v \leq 1$ a.e., where $v$ is given by (1.5).

The characterization of the set of all measures in $\mathcal{M}(\Omega)$ which are good for every $g$ will be given in Section 7 .

Our method in the study of problem (1.1) starts with a standard procedure which consists in approximating $g$ with bounded continuous functions defined on 
the whole $\mathbb{R}$. More precisely, let $\left(g_{n}\right)$ be a sequence of bounded functions $g_{n}: \mathbb{R} \rightarrow$ $\mathbb{R}$ which are continuous, nondecreasing and satisfy the following conditions:

$$
\begin{array}{ll}
0 \leq g_{1}(t) \leq g_{2}(t) \leq \ldots & \forall t \in \mathbb{R}, \\
g_{n}(t) \rightarrow g(t) & \forall t<1
\end{array}
$$

and

$$
g_{n}(t) \rightarrow+\infty \quad \forall t \geq 1 .
$$

Since each $g_{n}$ is bounded, there exists a unique solution $u_{n}$ of

$$
\left\{\begin{aligned}
-\Delta u_{n}+g_{n}\left(u_{n}\right)=\mu & & \text { in } \Omega, \\
u_{n}=0 & & \text { on } \partial \Omega .
\end{aligned}\right.
$$

Passing to the limit as $n$ tends to infinity we get the following result:

Proposition 1 Given any $\mu \in \mathcal{M}(\Omega)$, then $u_{n} \downarrow u^{*}$ in $\Omega$ as $n \uparrow+\infty$, where $u^{*}$ is the largest subsolution of (1.1). Moreover, we have

$$
\left\|g\left(u^{*}\right)\right\|_{L^{1}} \leq\|\mu\|_{\mathcal{M}}
$$

and

$$
\left|\int_{\Omega} u^{*} \Delta \zeta\right| \leq 2\|\mu\|_{\mathcal{M}}\|\zeta\|_{L^{\infty}} \quad \forall \zeta \in C_{0}^{2}(\bar{\Omega}) .
$$

Here, we denote by

$$
C_{0}^{2}(\bar{\Omega})=\left\{\zeta \in C^{2}(\bar{\Omega}): \zeta=0 \text { on } \partial \Omega\right\} .
$$

In the spirit of [4], we then define the reduced measure $\mu^{*}$ as

$$
\mu^{*}=-\Delta u^{*}+g\left(u^{*}\right)
$$

and we study the properties of $\mu^{*}$. First of all, since $u^{*}$ is the largest subsolution of $(1.1), \mu^{*}$ is well-defined, independently of the sequence $\left(g_{n}\right)$. Note that $\mu^{*} \leq \mu$; moreover, $\mu$ is a good measure if and only if $\mu=\mu^{*}$.

We have the following

Theorem 4 For every $\mu \in \mathcal{M}(\Omega)$, there exist Borel sets $\Sigma_{1}, \Sigma_{2} \subset \Omega$ such that

$$
\Sigma_{1} \subset\left[u^{*}=1\right], \quad \operatorname{cap}\left(\Sigma_{2}\right)=0, \quad \text { and } \quad\left(\mu-\mu^{*}\right)\left(\Omega \backslash\left(\Sigma_{1} \cup \Sigma_{2}\right)\right)=0 .
$$


Note that, in the previous statement, the set $\left[u^{*}=1\right]$ is well-defined up to sets of zero $H^{1}$-capacity. Indeed, any function $v \in L^{1}(\Omega)$ such that $\Delta v \in \mathcal{M}(\Omega)$ admits a unique cap-quasicontinuous representative $\tilde{v}$ (see e.g. [1]); henceforth, we shall always identify $v$ and $\tilde{v}$. We recall that $\tilde{v}$ is cap-quasicontinuous if, for every $\varepsilon>0$, there exists an open set $\omega_{\varepsilon} \subset \Omega$ such that $\operatorname{cap}\left(\omega_{\varepsilon}\right)<\varepsilon$ and $\left.\tilde{v}\right|_{\Omega \backslash \omega_{\varepsilon}}$ is continuous.

We remark that, in Theorem 4 , both sets $\Sigma_{1}$ and $\Sigma_{2}$ have zero Lebesgue measure, so that we deduce the following

Corollary 1 For any measure $\mu$, we have

$$
\left(\mu^{*}\right)_{\mathrm{a}}=\mu_{\mathrm{a}},
$$

where "a" denotes the absolutely continuous part with respect to the Lebesgue measure.

In view of Theorem 4 , if $\mu$ is diffuse and $\mu\left(\left[u^{*}=1\right]\right)=0$, then it follows that $\mu^{*}=\mu$, hence $\mu$ is good. We use this idea in order to prove Theorem 2 ; in this case the main effort is thus to estimate the $(N-2+\beta)$-Hausdorff measure of the set $\left[u^{*}=1\right]$. This kind of estimate, which has an interest in its own, is given by Theorem 12 in Section 4. In Section 5, we present another approach based on energy estimates; in this case, the "smallness" of $\left[u^{*}=1\right]$ is given in terms of (Sobolev) capacities.

The next result says that $\mu^{*}$ is the "best approximation" of $\mu$ in the class of good measures relative to $g$. More precisely,

Theorem 5 Given $\mu \in \mathcal{M}(\Omega)$, we have

$$
\left\|\mu-\mu^{*}\right\|_{\mathcal{M}}=\min _{\nu \in \mathcal{G}}\|\mu-\nu\|_{\mathcal{M}} .
$$

In addition, $\mu^{*}$ is the unique good measure for which the minimum in (1.13) is attained.

We recall that when the function $g$ is defined for every $t \in \mathbb{R}$, it has been shown in [4] that $\mu^{*}$ is the largest good measure $\leq \mu$. In that case, the characterization of $\mu^{*}$ given in Theorem 5 is then a straightforward consequence. We stress the following important difference in our case, namely there exist measures $\mu$ for which the set $\{\lambda \in \mathcal{G}(g): \lambda \leq \mu\}$ has no largest element (see Proposition 9 in Section 9). Thus, the fact that $\mu^{*}$ is the unique measure which achieves the minimum in (1.13) needs a direct proof, which is more delicate. 
Finally, two further differences with the case studied in [4] are worth being mentioned. When $g(t)$ is defined for every $t \in \mathbb{R}$, the set $\mathcal{G}$ of good measures is convex, and the mapping $\mu \mapsto \mu^{*}$ is a contraction. As we shall see in Section 9 below, these properties are no longer true when $g$ satisfies (1.2). In fact, for any such $g$ we have

(a) $\mathcal{G}$ is not convex;

(b) the mapping $\mu \mapsto \mu^{*}$ is not a contraction.

We would like to emphasize that throughout this paper we assume that $\Omega$ is a domain of $\mathbb{R}^{N}$, with $N \geq 2$. The case of dimension $N=1$ is different and has been studied by Vázquez [20]. We recall that in this case every measure is diffuse - since cap $(\{x\})>0$ for every $x$ - and the solutions of (1.1) are Lipschitz continuous. In [20], Vázquez proves that

$\left(a^{\prime}\right)$ if $\int_{0}^{1} g=+\infty$, then every $\mu \in \mathcal{M}(\Omega)$ is good;

$\left(b^{\prime}\right)$ if $\int_{0}^{1} g<+\infty$ and $\mu \in \mathcal{M}(\Omega)$ satisfies $\left\|\mu^{+}\right\|_{\mathcal{M}} \leq 2 \sqrt{2}\left(\int_{0}^{1} g\right)^{1 / 2}$, then $\mu$ is
good.

These two results have no counterpart when $N \geq 2$. According to Theorem 1 above, for any $g$ there exists a diffuse measure $\mu \geq 0$ such that $\mu$ is not good. As we shall see in Section 8, such $\mu$ can be chosen so that $\varepsilon \mu$ is not good for any $\varepsilon>0$.

The plan of this paper is the following:

1. Introduction;

2. Proofs of Proposition 1 and Theorem 4;

3. The reduced measure is the closest good measure;

4. Proof of Theorem 2;

5. Capacitary estimates related to problem (1.1);

6. Every diffuse measure is good for some $g$;

7. Measures which are good for every $g$; 
8. How to construct diffuse measures which are not good;

9. Further properties of $\mu^{*}$ and $\mathcal{G}$;

References.

\section{Proofs of Proposition 1 and Theorem 4}

We start by recalling that every measure $\mu$ can be uniquely decomposed as (see e.g. [16])

$$
\mu=\mu_{\mathrm{d}}+\mu_{\mathrm{c}},
$$

where $\mu_{\mathrm{d}}$ is diffuse and $\mu_{\mathrm{c}}$ is concentrated on a set of zero capacity. In particular, $\mu$ is diffuse if and only if $\mu_{\mathrm{c}}=0$.

A useful characterization of measures which are diffuse is given by the following

Theorem $6([\mathbf{3}, \mathbf{1 7}])$ Let $\mu \in \mathcal{M}(\Omega)$. Then, $\mu$ is diffuse if and only if

$$
\mu \in L^{1}(\Omega)+H^{-1}(\Omega) .
$$

The next two results will be often used in this paper:

Theorem 7 ([6]) Let $v \in L^{1}(\Omega)$ be such that $\Delta v \in \mathcal{M}(\Omega)$. Then, $\Delta v^{+} \in$ $\mathcal{M}_{\mathrm{loc}}(\Omega)$ and

$$
\begin{aligned}
\left(\Delta v^{+}\right)_{\mathrm{d}} & \geq \chi_{[v \geq 0]}(\Delta v)_{\mathrm{d}} & & \text { in } \Omega, \\
\left(-\Delta v^{+}\right)_{\mathrm{c}} & =(-\Delta v)_{\mathrm{c}}^{+} & & \text {in } \Omega .
\end{aligned}
$$

Moreover, if $v \geq 0$ a.e., then

$$
(\Delta v)_{\mathrm{d}} \geq 0 \quad \text { in }[v=0] .
$$

Theorem 8 ([14]) Let $v \in L^{1}(\Omega)$ be such that $\Delta v \in \mathcal{M}(\Omega)$. If $v \geq 0$ a.e., then

$$
(\Delta v)_{\mathrm{c}} \leq 0 \quad \text { in } \Omega
$$

As a result, we get a necessary condition in order that (1.1) admit a solution.

Corollary 2 If $\mu$ is good, then $\mu^{+}$is diffuse. 
Proof. Applying Theorem 8 to $v=1-u$ we get

$$
\mu_{\mathrm{c}}=(-\Delta u)_{\mathrm{c}}=(\Delta v)_{\mathrm{c}} \leq 0 .
$$

Thus, $\mu^{+}=\left(\mu_{\mathrm{d}}\right)^{+}=\left(\mu^{+}\right)_{\mathrm{d}}$ and so $\mu^{+}$is diffuse.

Let us also recall that, given $\nu \in \mathcal{M}(\Omega)$, there exists a unique function $v \in$ $L^{1}(\Omega)$ which satisfies

$$
-\int_{\Omega} v \Delta \zeta=\int_{\Omega} \zeta d \nu \quad \forall \zeta \in C_{0}^{2}(\bar{\Omega}) .
$$

This function is called Stampacchia's solution of the problem (see [19])

$$
\left\{\begin{aligned}
-\Delta v=\nu & \text { in } \Omega, \\
v=0 & \text { on } \partial \Omega,
\end{aligned}\right.
$$

and it coincides with the notion of "renormalized solution" introduced in [9]. In particular, Theorems 2.33 and 10.1 of [9] provide the following useful

Theorem 9 ([9]) Let $v$ be the unique solution of $(2.5)$. Let $\Phi \in W^{2, \infty}(\mathbb{R})$ be such that $\operatorname{supp} \Phi^{\prime \prime}$ is compact. Then, we have

$$
\Delta \Phi(v)=\Phi^{\prime}(v)(\Delta v)_{\mathrm{d}}+\Phi^{\prime \prime}(v)|\nabla v|^{2}-\Phi^{\prime}(+\infty)(\Delta v)_{\mathrm{c}}^{-}+\Phi^{\prime}(-\infty)(\Delta v)_{\mathrm{c}}^{+} \quad \text { in } \Omega .
$$

Here, we denote by $\Phi^{\prime}( \pm \infty)$ the limit of $\Phi^{\prime}$ as $|x| \rightarrow \pm \infty$.

The proof of Proposition 1 follows along the same lines as in [4]. Below, we present the proof for the convenience of the reader.

Proof of Proposition 1. Let $u_{n}$ be the solution of (1.9). Since $g_{n} \leq g_{n+1}$, by a comparison principle (see e.g. [4, Appendix B]) we have $u_{n} \geq u_{n+1}$. Hence, we define $u^{*}$ such that

$$
u_{n} \downarrow u^{*} \quad \text { a.e. in } \Omega .
$$

Standard estimates imply that

$$
\left\|g_{n}\left(u_{n}\right)\right\|_{L^{1}} \leq\|\mu\|_{\mathcal{M}}
$$

thus,

$$
\left|\int_{\Omega} u_{n} \Delta \zeta\right|=\left|\int_{\Omega} \zeta d \mu-\int_{\Omega} g_{n}\left(u_{n}\right) \zeta\right| \leq 2\|\mu\|_{\mathcal{M}}\|\zeta\|_{L^{\infty}} \quad \forall \zeta \in C_{0}^{2}(\bar{\Omega}) .
$$

Clearly, $u^{*} \in L^{1}(\Omega)$ and $\left(u_{n}\right)$ converges strongly to $u^{*}$ in $L^{1}(\Omega)$. Moreover, it follows from (2.6) that $u^{*} \leq 1$ a.e. Then, by using Fatou's lemma, we deduce from the previous estimates that 
(i) $g\left(u^{*}\right) \in L^{1}(\Omega)$ and (1.10) holds;

(ii) $\Delta u^{*} \in \mathcal{M}(\Omega)$ and $\left\|\Delta u^{*}\right\|_{\mathcal{M}} \leq 2\|\mu\|_{\mathcal{M}}$.

Finally, let $v$ be any subsolution of (1.1), i.e. $v \in L^{1}(\Omega), v \leq 1$ a.e., $g(v) \in L^{1}(\Omega)$ and

$$
-\int_{\Omega} v \Delta \zeta+\int_{\Omega} g(v) \zeta \leq \int_{\Omega} \zeta d \mu \quad \forall \zeta \in C_{0}^{2}(\bar{\Omega}), \zeta \geq 0 \text { in } \Omega .
$$

Since $g_{n} \leq g$, we have

$$
-\Delta v+g_{n}(v) \leq-\Delta v+g(v) \leq \mu=-\Delta u_{n}+g_{n}\left(u_{n}\right) \quad \text { in }\left[C_{0}^{2}(\bar{\Omega})\right]^{*},
$$

which yields $v \leq u_{n}$ a.e. Passing to the limit, we deduce that $v \leq u^{*}$. This proves that $u^{*}$ is the largest subsolution of (1.1).

Let

$$
\mu^{*}=-\Delta u^{*}+g\left(u^{*}\right) \quad \text { in } \mathcal{D}^{\prime}(\Omega) .
$$

In view of Proposition $1, \mu^{*} \in \mathcal{M}(\Omega)$. The reduced measure $\mu^{*}$ is uniquely determined by the weak ${ }^{*}$ limit of $g_{n}\left(u_{n}\right)$ in $\mathcal{M}(\Omega)$. Indeed, comparing (2.7) with (1.9), and using that $u_{n} \rightarrow u^{*}$ in $L^{1}(\Omega)$, we obtain the following

Lemma 1 Let $\left(u_{n}\right)$ be the sequence defined in Proposition 1. Then,

$$
g_{n}\left(u_{n}\right) \stackrel{*}{\rightarrow} g\left(u^{*}\right)+\left(\mu-\mu^{*}\right) \quad \text { weak } k^{*} \text { in } \mathcal{M}(\Omega) .
$$

Note that, since $\Delta u^{*} \in \mathcal{M}(\Omega)$, the function $u^{*}$ admits a unique cap-quasicontinuous representative, which we are going to use henceforth as our standard choice. In particular, we remark that the set $\left[u^{*}=1\right]$ is uniquely defined up to sets of zero capacity.

The main ingredient in the proof of Theorem 4 is the next

Proposition 2 Let $u^{*}$ be given by Proposition 1 and let $\mu^{*}$ be the reduced measure defined in (2.7). Then, we have

$$
0 \leq \mu-\mu^{*} \leq\left(\mu_{\mathrm{d}}\right)\left\lfloor_{\left[u^{*}=1\right]}+\mu_{\mathrm{c}}^{+} \quad \text { in } \Omega .\right.
$$

In particular,

$$
\begin{cases}\left(\mu^{*}\right)_{\mathrm{d}}=\mu_{\mathrm{d}} & \text { in }\left[u^{*}<1\right], \\ \left(\mu^{*}\right)_{\mathrm{d}} \geq 0 & \text { in }\left[u^{*}=1\right], \\ \left(\mu^{*}\right)_{\mathrm{c}}=-\left(\mu_{\mathrm{c}}\right)^{-} & \text {in } \Omega, \\ \left(\mu^{*}\right)^{-}=\mu^{-} & \text {in } \Omega .\end{cases}
$$




\section{Proof.}

Step 1. Proof of (2.9).

Given $\delta>0$, let us define the function $\theta_{\delta}(s)=\min \left\{1, \frac{1}{\delta}(s-1+2 \delta)^{+}\right\}$. Applying Theorem 9 with $v=u_{n}$ and $\Phi_{\delta}(s)=\int_{0}^{s} \theta_{\delta}(\xi) d \xi$ we get, for any $\zeta \in C_{0}^{2}(\bar{\Omega})$, $\zeta \geq 0$ in $\Omega$,

$$
\int_{\Omega} g_{n}\left(u_{n}\right) \theta_{\delta}\left(u_{n}\right) \zeta d x \leq \int_{\Omega} \theta_{\delta}\left(u_{n}\right) \zeta d \mu_{\mathrm{d}}+\int_{\Omega} \zeta d \mu_{\mathrm{c}}^{+}+\int_{\Omega} \Phi_{\delta}\left(u_{n}\right) \Delta \zeta d x,
$$

which yields

$$
\int_{\left[u_{n}>1-\delta\right]} g_{n}\left(u_{n}\right) \zeta d x \leq \int_{\Omega} \theta_{\delta}\left(u_{n}\right) \zeta d \mu_{\mathrm{d}}+\int_{\Omega} \zeta d \mu_{\mathrm{c}}^{+}+\int_{\Omega} \Phi_{\delta}\left(u_{n}\right) \Delta \zeta d x .
$$

Since $\left(u_{n}^{+}\right)$is bounded in $L^{\infty}(\Omega)$ and $\left(\Delta u_{n}\right)$ is bounded in $\mathcal{M}(\Omega)$, the sequence $\left(\theta_{\delta}\left(u_{n}\right)\right)$ is bounded in $H_{0}^{1}(\Omega)$, converges weakly to $\theta_{\delta}\left(u^{*}\right)$ in $H_{0}^{1}(\Omega)$ and weak* in $L^{\infty}(\Omega)$. Moreover, since $\mu_{\mathrm{d}} \in L^{1}(\Omega)+H^{-1}(\Omega)$ (by Theorem 6 ), we have

$$
\lim _{n \rightarrow+\infty} \int_{\Omega} \theta_{\delta}\left(u_{n}\right) \zeta d \mu_{\mathrm{d}}=\int_{\Omega} \theta_{\delta}\left(u^{*}\right) \zeta d \mu_{\mathrm{d}}
$$

Thus,

$$
\limsup _{n \rightarrow+\infty} \int_{\left[u_{n}>1-\delta\right]} g_{n}\left(u_{n}\right) \zeta d x \leq \int_{\Omega} \theta_{\delta}\left(u^{*}\right) \zeta d \mu_{\mathrm{d}}+\int_{\Omega} \zeta d \mu_{\mathrm{c}}^{+}+\int_{\Omega} \Phi_{\delta}\left(u^{*}\right) \Delta \zeta d x .
$$

Clearly, by dominated convergence we have, for a.e. $\delta>0$,

$$
\lim _{n \rightarrow+\infty} \int_{\left[u_{n} \leq 1-\delta\right]} g_{n}\left(u_{n}\right) \zeta d x=\int_{\left[u^{*} \leq 1-\delta\right]} g\left(u^{*}\right) \zeta d x .
$$

Therefore,

$$
\begin{aligned}
\limsup _{n \rightarrow+\infty} & \int_{\Omega} g_{n}\left(u_{n}\right) \zeta d x \leq \\
& \leq \int_{\left[u^{*} \leq 1-\delta\right]} g\left(u^{*}\right) \zeta d x+\int_{\Omega} \theta_{\delta}\left(u^{*}\right) \zeta d \mu_{\mathrm{d}}+\int_{\Omega} \zeta d \mu_{\mathrm{c}}^{+}+\int_{\Omega} \Phi_{\delta}\left(u^{*}\right) \Delta \zeta d x,
\end{aligned}
$$

for a.e. $\delta>0$. Since $u^{*}<1$ a.e., we have $\Phi_{\delta}\left(u^{*}\right) \rightarrow 0$ a.e. By dominated convergence, as $\delta \rightarrow 0$ we obtain

$$
\limsup _{n \rightarrow+\infty} \int_{\Omega} g_{n}\left(u_{n}\right) \zeta d x \leq \int_{\Omega} g\left(u^{*}\right) \zeta d x+\int_{\left[u^{*}=1\right]} \zeta d \mu_{\mathrm{d}}+\int_{\Omega} \zeta d \mu_{\mathrm{c}}^{+} .
$$


Comparing with (2.8) we get

$$
\mu-\mu^{*} \leq\left(\mu_{\mathrm{d}}\right)\left\lfloor_{\left[u^{*}=1\right]}+\mu_{\mathrm{c}}^{+} .\right.
$$

Clearly, by Fatou's lemma, $\mu^{*} \leq \mu$. We thus obtain (2.9).

Step 2. Proof of (2.10).

From (2.9) we immediately deduce that

$$
\begin{array}{ll}
\left(\mu^{*}\right)_{\mathrm{d}}=\mu_{\mathrm{d}} & \text { in }\left[u^{*}<1\right], \\
\left(\mu^{*}\right)_{\mathrm{d}} \geq 0 & \text { in }\left[u^{*}=1\right] .
\end{array}
$$

Since $\mu_{\mathrm{d}} \geq\left(\mu^{*}\right)_{\mathrm{d}},(2.12)$ yields

$$
\left(\mu_{\mathrm{d}}\right)^{-}=\left(\mu^{*}\right)_{\mathrm{d}}^{-} \quad \text { in } \Omega .
$$

On the other hand, by (2.9),

$$
\mu_{\mathrm{c}}-\left(\mu^{*}\right)_{\mathrm{c}} \leq \mu_{\mathrm{c}}^{+} \quad \text { in } \Omega
$$

that is

$$
\left(\mu^{*}\right)_{\mathrm{c}} \geq-\mu_{\mathrm{c}}^{-} \quad \text { in } \Omega .
$$

Note that $\mu^{*} \leq \mu$ and $\left(\mu^{*}\right)_{\mathrm{c}} \leq 0$ (by Corollary 2 ); thus,

$$
\left(\mu^{*}\right)_{\mathrm{c}} \leq-\mu_{\mathrm{c}}^{-} \quad \text { in } \Omega .
$$

We deduce that

$$
\left(\mu^{*}\right)_{\mathrm{c}}=-\mu_{\mathrm{c}}^{-} \quad \text { in } \Omega .
$$

Assertion (2.10) then follows from (2.12)-(2.14).

As a consequence of the previous result we have the

Proof of Theorem 4. Let $\Sigma_{1}=\left[u^{*}=1\right]$ and let $\Sigma_{2} \subset \Omega$ be such that cap $\left(\Sigma_{2}\right)=$ 0 and $\mu_{\mathrm{c}}^{+}\left(\Omega \backslash \Sigma_{2}\right)=0$. With this choice, (1.12) follows immediately from (2.9).

As a corollary of (2.10) we also have the

Corollary 3 Let $\mu \in \mathcal{M}(\Omega)$. If $\mu \geq 0$, then $\mu^{*} \geq 0$. 
We give an alternative characterization of $u^{*}$ in the next

Proposition 3 For every $\mu \in \mathcal{M}(\Omega)$, $u^{*}$ is the unique solution of the following problem:

$$
\left\{\begin{aligned}
v \in W_{0}^{1,1}(\Omega), \quad v \leq 1 & \text { a.e., } \quad \Delta v \in \mathcal{M}(\Omega), \quad g(v) \in L^{1}(\Omega), \\
(-\Delta v)_{\mathrm{d}}+g(v)=\mu_{\mathrm{d}} & \text { in }[v<1], \\
(-\Delta v)_{\mathrm{d}} \leq \mu_{\mathrm{d}} & \text { in }[v=1], \\
(-\Delta v)_{\mathrm{c}}=-\mu_{\mathrm{c}}^{-} & \text {in } \Omega .
\end{aligned}\right.
$$

Proof. From (2.7) and (2.10), it follows that $u^{*}$ is indeed a solution of (2.15). We now prove that the solution of (2.15) is unique. Assume that $v_{1}, v_{2}$ both satisfy (2.15) and consider the function $w=\left(v_{1}-v_{2}\right)^{+}$. We first observe that $\Delta w$ is a measure and, by (2.3),

$$
(\Delta w)_{\mathrm{d}} \geq 0 \quad \text { in }\left[v_{1} \leq v_{2}\right] .
$$

By (2.15),

$$
\left(\Delta v_{1}\right)_{\mathrm{d}} \geq g\left(v_{1}\right)-\mu_{\mathrm{d}} \quad \text { in } \Omega .
$$

On the other hand, since $\left[v_{1}>v_{2}\right] \subset\left[v_{2}<1\right]$, we have

$$
\left(\Delta v_{2}\right)_{\mathrm{d}}=g\left(v_{2}\right)-\mu_{\mathrm{d}} \quad \text { in }\left[v_{1}>v_{2}\right] .
$$

Thus, by (2.17)-(2.18),

$$
(\Delta w)_{\mathrm{d}} \geq\left[\Delta\left(v_{1}-v_{2}\right)\right]_{\mathrm{d}} \geq g\left(v_{1}\right)-g\left(v_{2}\right) \geq 0 \quad \text { in }\left[v_{1}>v_{2}\right] .
$$

We deduce from (2.16) and (2.19) that

$$
(\Delta w)_{\mathrm{d}} \geq 0 \text { in } \Omega .
$$

Since, by $(2.2)$,

$$
(-\Delta w)_{\mathrm{c}}=\left[-\Delta\left(v_{1}-v_{2}\right)\right]_{\mathrm{c}}^{+} \text {in } \Omega,
$$

we get

$$
(-\Delta w)_{\mathrm{c}}=\left[\left(-\Delta v_{1}\right)_{\mathrm{c}}+\left(\Delta v_{2}\right)_{\mathrm{c}}\right]^{+}=0 \text { in } \Omega .
$$

From (2.20)-(2.21) we obtain that

$$
\Delta w \geq 0 \quad \text { in } \Omega .
$$

Since $w$ vanishes on $\partial \Omega$, we have $v_{1} \leq v_{2}$ a.e. in $\Omega$ (see e.g. [4, Proposition B.1]). Reversing the roles of the two functions we finally obtain that $v_{1}=v_{2}$. 
Until now, we have studied problem (1.1) by approximating the nonlinearity $g$ using a sequence $\left(g_{n}\right)$, with $\mu$ fixed. Another possible approach is to fix $g$ and to approximate $\mu$ by $\rho_{n} * \mu$, where $\left(\rho_{n}\right)$ is a sequence of mollifiers. More precisely, $\rho_{n} * \mu$ is given by

$$
\left(\rho_{n} * \mu\right)(x)=\int_{\Omega} \rho_{n}(x-y) d \mu(y) \quad \forall x \in \Omega .
$$

It turns out that the sequences of solutions in both cases converge to the same limit. More precisely,

Theorem 10 Let $\mu \in \mathcal{M}(\Omega)$. For each $n \geq 1$, let $v_{n}$ be the solution of

$$
\left\{\begin{aligned}
-\Delta v_{n}+g\left(v_{n}\right) & =\rho_{n} * \mu & & \text { in } \Omega, \\
v_{n} & =0 & & \text { on } \partial \Omega .
\end{aligned}\right.
$$

Then, $v_{n} \rightarrow u^{*}$ in $L^{1}(\Omega)$, where $u^{*}$ is the function given by Proposition 1 .

Proof. By standard estimates we have

$$
\left\|g\left(v_{n}\right)\right\|_{L^{1}(\Omega)} \leq\left\|\rho_{n} * \mu\right\|_{\mathcal{M}(\Omega)} \leq\|\mu\|_{\mathcal{M}(\Omega)}
$$

Thus, $\Delta v_{n}$ is bounded in $L^{1}(\Omega)$ and there exist $v \in L^{1}(\Omega)$ and $\nu \in \mathcal{M}(\Omega)$ such that, for a subsequence (still denoted $\left(v_{n}\right)$ ), we have

$$
\begin{aligned}
& v_{n} \rightarrow v \text { strongly in } L^{1}(\Omega) \text { and a.e. } \\
& g\left(v_{n}\right) \stackrel{*}{\rightarrow} g(v)+\nu \quad \text { weak }^{*} \text { in } \mathcal{M}(\Omega) .
\end{aligned}
$$

By Fatou's lemma, we have $\nu \geq 0$. Moreover, it follows that $v$ satisfies

$$
-\Delta v+g(v)=\mu-\nu \text { in } \Omega .
$$

We now follow the outline of the proof of Proposition 2. Take $\theta_{\delta}\left(v_{n}\right) \zeta$ as a test function in $(2.22)$, where $\zeta \in C_{0}^{2}(\bar{\Omega})$. We get the analog of $(2.11)$, namely

$\int_{\Omega} g\left(v_{n}\right) \theta_{\delta}\left(v_{n}\right) \zeta d x \leq \int_{\Omega} \theta_{\delta}\left(v_{n}\right)\left(\rho_{n} * \mu_{\mathrm{d}}\right) \zeta d x+\int_{\Omega}\left(\rho_{n} * \mu_{\mathrm{c}}^{+}\right) \zeta d x+\int_{\Omega} \Phi_{\delta}\left(v_{n}\right) \Delta \zeta d x$.

As in Step 1 of Proposition 2 we obtain, as $n$ tends to infinity, that

$$
\nu \leq\left(\mu_{\mathrm{d}}\right)\left\lfloor_{[v=1]}+\mu_{\mathrm{c}}^{+} \quad \text { in } \Omega .\right.
$$

Thanks to (2.23)-(2.24), we obtain that $v$ is a solution of (2.15) (see Step 2 of Proposition 2). From the uniqueness result of Proposition 3, we conclude that $v=u^{*}$. In particular, the whole sequence $\left(v_{n}\right)$ converges to $u^{*}$. 


\section{The reduced measure is the closest good mea- sure}

We start with the following simple result:

Proposition 4 Let $\mu \in \mathcal{M}(\Omega)$. If $\mu \in \mathcal{G}(g)$ and $\nu \leq \mu$, then $\nu \in \mathcal{G}(g)$.

Proof. Let $\left(u_{n}\right)$ be the sequence of functions satisfying (1.9). By standard estimates (see e.g. [4]), we have

$$
\int_{\Omega}\left|g_{n}\left(u_{n}\right)-g_{n}(u)\right| \leq \int_{\Omega}\left|g(u)-g_{n}(u)\right| .
$$

Thus,

$$
\int_{\Omega}\left|g_{n}\left(u_{n}\right)-g(u)\right| \leq 2 \int_{\Omega}\left|g(u)-g_{n}(u)\right| \rightarrow 0 \quad \text { as } n \rightarrow+\infty .
$$

We conclude that $g_{n}\left(u_{n}\right) \rightarrow g(u)$ in $L^{1}(\Omega)$. Let $\left(v_{n}\right)$ be the sequence associated to $\nu$. By comparison, $\nu \leq \mu$ implies $v_{n} \leq u_{n}$ a.e.; thus, $g_{n}\left(v_{n}\right) \leq g_{n}\left(u_{n}\right)$ a.e. Applying the Dominated convergence theorem, we conclude that $g_{n}\left(v_{n}\right) \rightarrow g\left(v^{*}\right)$ in $L^{1}(\Omega)$. We then deduce that $v^{*}$ is the solution of (1.1) with data $\nu$, and so $\nu$ is a good measure.

We also have the following

Lemma 2 For every $\mu, \nu \in \mathcal{M}(\Omega)$, we have

$$
\int_{\Omega}\left|g\left(u^{*}\right)-g\left(v^{*}\right)\right|+\left\|\left(\mu-\mu^{*}\right)-\left(\nu-\nu^{*}\right)\right\|_{\mathcal{M}} \leq\|\mu-\nu\|_{\mathcal{M}},
$$

where $u^{*}, v^{*}$ are the solutions of (1.1) with respect to $\mu^{*}, \nu^{*}$, resp.

Proof. Let $v_{n}$ denote the solution of

$$
\left\{\begin{aligned}
-\Delta v_{n}+g_{n}\left(v_{n}\right)=\nu & & \text { in } \Omega, \\
v_{n}=0 & & \text { on } \partial \Omega .
\end{aligned}\right.
$$

By Lemma 1, we get

$$
g_{n}\left(u_{n}\right)-g_{n}\left(v_{n}\right) \stackrel{*}{\rightarrow} g\left(u^{*}\right)-g\left(v^{*}\right)+\left(\mu-\mu^{*}\right)-\left(\nu-\nu^{*}\right) \quad \text { weak }^{*} \text { in } \mathcal{M}(\Omega) .
$$

Since $\mu-\mu^{*}$ and $\nu-\nu^{*}$ are both singular with respect to the Lebesgue measure (see Corollary 1), we have

$\left\|g\left(u^{*}\right)-g\left(v^{*}\right)+\left(\mu-\mu^{*}\right)-\left(\nu-\nu^{*}\right)\right\|_{\mathcal{M}}=\int_{\Omega}\left|g\left(u^{*}\right)-g\left(v^{*}\right)\right|+\left\|\left(\mu-\mu^{*}\right)-\left(\nu-\nu^{*}\right)\right\|_{\mathcal{M}}$. 
On the other hand,

$$
\left\|g_{n}\left(u_{n}\right)-g_{n}\left(v_{n}\right)\right\|_{L^{1}} \leq\|\mu-\nu\|_{\mathcal{M}} \quad \forall n \geq 1 .
$$

Thus,

$$
\begin{aligned}
\int_{\Omega}\left|g\left(u^{*}\right)-g\left(v^{*}\right)\right|+\left\|\left(\mu-\mu^{*}\right)-\left(\nu-\nu^{*}\right)\right\|_{\mathcal{M}} & \leq \liminf _{n \rightarrow+\infty}\left\|g_{n}\left(u_{n}\right)-g_{n}\left(v_{n}\right)\right\|_{L^{1}} \\
& \leq\|\mu-\nu\|_{\mathcal{M}},
\end{aligned}
$$

which gives (3.1).

Our next result is the following

Theorem 11 For every $\mu, \nu \in \mathcal{M}(\Omega)$, we have

$$
\left\|\mu^{*}-\nu^{*}\right\|_{\mathcal{M}} \leq 2\|\mu-\nu\|_{\mathcal{M}}
$$

Proof. Let $\mu, \nu \in \mathcal{M}(\Omega)$. By Lemma 2, we have

$$
\left\|\left(\mu-\mu^{*}\right)-\left(\nu-\nu^{*}\right)\right\|_{\mathcal{M}} \leq\|\mu-\nu\|_{\mathcal{M}}
$$

Applying the triangle inequality, we obtain (3.2).

We now present the

Proof of Theorem 5. We shall split the proof into two steps.

Step 1. Proof of (1.13).

Given $\nu \in \mathcal{G}$, we have $\nu=\nu^{*}$. It then follows from Lemma 2 that

$$
\int_{\Omega}\left|g\left(u^{*}\right)-g(v)\right|+\left\|\mu-\mu^{*}\right\|_{\mathcal{M}} \leq\|\mu-\nu\|_{\mathcal{M}}
$$

where $v$ is the solution of (1.1) with measure $\nu$. In particular,

$$
\left\|\mu-\mu^{*}\right\|_{\mathcal{M}} \leq\|\mu-\nu\|_{\mathcal{M}},
$$

which gives (1.13).

Step 2. $\mu^{*}$ is the unique good measure which achieves the minimum in (1.13).

We now assume that $\nu \in \mathcal{G}$ satisfies

$$
\|\mu-\nu\|_{\mathcal{M}}=\left\|\mu-\mu^{*}\right\|_{\mathcal{M}}
$$


By (3.3), we have

$$
\int_{\Omega}\left|g\left(u^{*}\right)-g(v)\right|=0
$$

Thus,

$$
g\left(u^{*}\right)=g(v) \quad \text { a.e. }
$$

We next observe that $\nu \leq \mu$. In fact, note that

$$
\inf \{\mu, \nu\}=\mu-(\mu-\nu)^{+} .
$$

Moreover, due to Proposition 4, inf $\{\mu, \nu\} \leq \nu \operatorname{implies~that~inf~}\{\mu, \nu\}$ is also a good measure. It then follows from (3.6) and the minimality of $\nu$ that

$$
\|\mu-\nu\|_{\mathcal{M}} \leq\|\mu-\inf \{\mu, \nu\}\|_{\mathcal{M}}=\left\|(\mu-\nu)^{+}\right\|_{\mathcal{M}} .
$$

Therefore, $(\mu-\nu)^{-}=0$; in other words, $\nu \leq \mu$. In particular, $v$ is a subsolution of (1.1), so that $v \leq u^{*}$ a.e. by Proposition 1 .

We now split the proof into two cases:

Case 1. $\operatorname{cap}\left(\left[u^{*}=1\right]\right)=0$.

By Theorem 4 , this implies $\left(\mu-\mu^{*}\right)_{\mathrm{d}}=0$. Thus,

$$
\nu_{\mathrm{d}} \leq \mu_{\mathrm{d}}=\left(\mu^{*}\right)_{\mathrm{d}}
$$

On the other hand, since $v \leq u^{*}$ a.e., it follows from Theorem 8 that

$$
\nu_{\mathrm{c}}=(-\Delta v)_{\mathrm{c}} \leq\left(-\Delta u^{*}\right)_{\mathrm{c}}=\left(\mu^{*}\right)_{\mathrm{c}} .
$$

We conclude that

$$
\nu \leq \mu^{*} \leq \mu
$$

By (3.4), we must have $\nu=\mu^{*}$.

Case 2. $\operatorname{cap}\left(\left[u^{*}=1\right]\right)>0$.

We first show that $u^{*}=v$ on a set of positive Lebesgue measure. By contradiction, suppose that $v<u^{*}$ a.e. Let $\alpha_{0}, \beta_{0} \in[0,1]$ be such that $\alpha_{0}<\beta_{0}$ and $g$ is increasing on $\left[\alpha_{0}, \beta_{0}\right]$. Since (3.5) holds and $v<u^{*}$ a.e., the set $\left[\alpha_{0}<u^{*}<\beta_{0}\right]$ has zero Lebesgue measure. Let

$$
w=\min \left\{\beta_{0}, \max \left\{\alpha_{0}, u^{*}\right\}\right\}-\alpha_{0} .
$$

Thus, $w \in H_{0}^{1}(\Omega)$ and $w$ achieves only the values 0 and $\beta_{0}-\alpha_{0}$. We conclude that $w=0$ a.e. In other words, $u^{*} \leq \alpha_{0}$ a.e. Since cap $\left(\left[u^{*}=1\right]\right)>0$, we get a contradiction. 
We now proceed with the proof of Case 2. Given $\varepsilon>0$, let $\alpha, \beta \in(1-\varepsilon, 1)$, $\alpha<\beta$, be such that $g$ is increasing in $[\alpha, \beta]$. Let $\Phi_{\varepsilon}: \mathbb{R} \rightarrow \mathbb{R}$ be a smooth function such that $\Phi_{\varepsilon}(t)=t$ if $t \leq \alpha, \Phi_{\varepsilon}(t)=1$ if $t \geq \beta$ and $\Phi^{\prime}(t) \geq 0, \forall t \in \mathbb{R}$. We now establish the following

Claim. For every $\varepsilon>0$, we have

$$
-\Delta\left[\Phi_{\varepsilon}\left(u^{*}\right)-\Phi_{\varepsilon}(v)\right] \geq 0 \quad \text { in } \mathcal{D}^{\prime}(\Omega) .
$$

In fact, by Theorem 9 ,

$$
\begin{aligned}
{\left[\Delta \Phi_{\varepsilon}\left(u^{*}\right)\right]_{\mathrm{d}} } & =\Phi_{\varepsilon}^{\prime}\left(u^{*}\right)\left(\Delta u^{*}\right)_{\mathrm{d}}+\Phi_{\varepsilon}^{\prime \prime}\left(u^{*}\right)\left|\nabla u^{*}\right|^{2} \\
& =\Phi_{\varepsilon}^{\prime}\left(u^{*}\right)\left[g\left(u^{*}\right)-\left(\mu^{*}\right)_{\mathrm{d}}\right]+\Phi_{\varepsilon}^{\prime \prime}\left(u^{*}\right)\left|\nabla u^{*}\right|^{2}
\end{aligned}
$$

and, similarly,

$$
\left[\Delta \Phi_{\varepsilon}(v)\right]_{\mathrm{d}}=\Phi_{\varepsilon}^{\prime}(v)\left[g(v)-\nu_{\mathrm{d}}\right]+\Phi_{\varepsilon}^{\prime \prime}(v)|\nabla v|^{2} .
$$

By construction of $\Phi_{\varepsilon}$, we have

$$
\Phi_{\varepsilon}^{\prime}\left(u^{*}\right)=\Phi_{\varepsilon}^{\prime}(v) \quad \text { a.e. }
$$

This is clear if $v \leq u^{*} \leq \alpha$ or $\beta \leq v \leq u^{*}$. Finally, if $\alpha<u^{*}$ and $v<\beta$, then $u^{*}=v$ a.e. since $g$ is increasing in $[\alpha, \beta]$ and $g\left(u^{*}\right)=g(v)$ a.e. We conclude that (3.10) holds.

By (3.5) and (3.10) we then have

$$
\Phi_{\varepsilon}^{\prime}\left(u^{*}\right) g\left(u^{*}\right)-\Phi_{\varepsilon}^{\prime}(v) g(v)=0 \quad \text { a.e. }
$$

Note that

$$
\Phi_{\varepsilon}^{\prime \prime}\left(u^{*}\right)=\Phi_{\varepsilon}^{\prime \prime}(v) \quad \text { a.e. }
$$

In addition, on the set where $\Phi_{\varepsilon}^{\prime \prime}\left(u^{*}\right) \neq 0$, we have $u^{*}=v$ a.e., so that

$$
\nabla u^{*}=\nabla v \quad \text { a.e. in }\left[\Phi_{\varepsilon}^{\prime \prime}\left(u^{*}\right) \neq 0\right] .
$$

Thus,

$$
\Phi_{\varepsilon}^{\prime \prime}\left(u^{*}\right)\left|\nabla u^{*}\right|^{2}-\Phi_{\varepsilon}^{\prime \prime}(v)|\nabla v|^{2}=0 \quad \text { a.e. }
$$

Finally, since $\Phi_{\varepsilon}^{\prime}(1)=0$ and $\left(\mu^{*}\right)_{\mathrm{d}}=\mu_{\mathrm{d}}$ on the set $\left[u^{*}<1\right]$ (by Theorem 4 ), we have

$$
\Phi_{\varepsilon}^{\prime}\left(u^{*}\right)\left(\mu^{*}\right)_{\mathrm{d}}=\Phi_{\varepsilon}^{\prime}\left(u^{*}\right) \mu_{\mathrm{d}} \quad \text { in } \Omega .
$$


Moreover, $\Phi_{\varepsilon}^{\prime} \geq 0$ and $\nu \leq \mu$ imply

$$
\Phi_{\varepsilon}^{\prime}(v) \nu_{\mathrm{d}} \leq \Phi_{\varepsilon}^{\prime}(v) \mu_{\mathrm{d}} \quad \text { in } \Omega .
$$

Therefore,

$$
\Phi_{\varepsilon}^{\prime}\left(u^{*}\right)\left(\mu^{*}\right)_{\mathrm{d}}-\Phi_{\varepsilon}^{\prime}(v) \nu_{\mathrm{d}} \geq\left[\Phi_{\varepsilon}^{\prime}\left(u^{*}\right)-\Phi_{\varepsilon}^{\prime}(v)\right] \mu_{\mathrm{d}}=0 \quad \text { in } \Omega .
$$

Subtracting (3.9) from (3.8), and then applying (3.11)-(3.13), we conclude that

$$
-\left(\Delta\left[\Phi_{\varepsilon}\left(u^{*}\right)-\Phi_{\varepsilon}(v)\right]\right)_{\mathrm{d}} \geq 0 \text { in } \Omega .
$$

On the other hand, since $u^{*} \geq v$ a.e., we have $\Phi_{\varepsilon}\left(u^{*}\right)-\Phi_{\varepsilon}(v) \geq 0$ a.e. It then follows from Theorem 8 that

$$
-\left(\Delta\left[\Phi_{\varepsilon}\left(u^{*}\right)-\Phi_{\varepsilon}(v)\right]\right)_{\mathrm{c}} \geq 0 \text { in } \Omega .
$$

Combining (3.14) and (3.15), we obtain (3.7). This concludes the proof of the claim.

According to the previous claim, the function $\Phi_{\varepsilon}\left(u^{*}\right)-\Phi_{\varepsilon}(v)$ is superharmonic. Moreover, since it is nonnegative and $\Phi_{\varepsilon}\left(u^{*}\right)=\Phi_{\varepsilon}(v)$ a.e. on a set of positive (Lebesgue) measure, we deduce from the strong maximum principle (see [1]; see also [5]) that

$$
\Phi_{\varepsilon}\left(u^{*}\right)=\Phi_{\varepsilon}(v) \quad \text { a.e. in } \Omega .
$$

Since this holds true for every $\varepsilon>0$, as we let $\varepsilon \downarrow 0$ we conclude that $u^{*}=v$ a.e. Thus, $\mu^{*}=\nu$. The proof of Theorem 5 is complete.

\section{Proof of Theorem 2}

In order to establish Theorem 2, we shall assume the next result which will be proved afterwards:

Theorem 12 Let $v \in L^{1}(\Omega), v \leq 1$ a.e., be such that $\Delta v \in \mathcal{M}(\Omega)$. Assume $g$ satisfies (1.4) for some $0<\beta<2$. If $g(v) \in L^{1}(\Omega)$, then

$$
\mathcal{H}^{N-2+\beta}([v=1])=0 .
$$


Proof of Theorem 2. Clearly, it suffices to establish the theorem for $\mu \geq 0$. Let $u^{*}$ be the function given by Proposition 1 . Since $\Delta u^{*} \in \mathcal{M}(\Omega)$ and $g\left(u^{*}\right) \in L^{1}(\Omega)$, it follows from Theorem 12 that

$$
\mathcal{H}^{N-2+\beta}\left(\left[u^{*}=1\right]\right)=0 .
$$

By assumption, we have $\mu \ll \mathcal{H}^{N-2+\beta}$. Thus, $\mu$ is diffuse and

$$
\mu\left(\left[u^{*}=1\right]\right)=0 .
$$

We deduce from Theorem 4 that $\mu^{*}=\mu$. In other words, $\mu \in \mathcal{G}$. This concludes the proof of Theorem 2.

We shall split the proof of Theorem 12 into two cases, whether $0<\beta<1$ or $1 \leq \beta<2$. We first consider the case where $0<\beta<1$. An important ingredient is the following

Lemma 3 Let $\nu \in \mathcal{M}(\Omega)$ and let $v$ be the solution of

$$
\left\{\begin{aligned}
-\Delta v=\nu & \text { in } \Omega \\
v=0 & \text { on } \partial \Omega
\end{aligned}\right.
$$

Given $0<\beta<1$ and $k \geq 1$, there exists a Borel set $A_{k} \subset \Omega$ such that

$$
|v(x)-v(y)| \leq C k|x-y|^{\beta} \quad \forall x, y \in \Omega \backslash A_{k}
$$

and

$$
\mathcal{H}_{\infty}^{N-2+\beta}\left(A_{k}\right) \leq \frac{C}{k}\|\nu\|_{\mathcal{M}}
$$

for some constant $C>0$ independent of $k$.

Given $\alpha \geq 0$, the Hausdorff content $\mathcal{H}_{\infty}^{\alpha}$ of a Borel set $A \subset \mathbb{R}^{N}$ is defined as

$$
\mathcal{H}_{\infty}^{\alpha}(A)=\inf \left\{\sum_{i} r_{i}^{\alpha}: A \subset \bigcup_{i} B_{r_{i}}\left(x_{i}\right)\right\},
$$

where the infimum is taken over all coverings of $A$ with balls $B_{r_{i}}\left(x_{i}\right)$ of radii $r_{i}$. Note that we make no restriction on the size of such balls. In particular, for every bounded set $A$ we have $\mathcal{H}_{\infty}^{\alpha}(A)<\infty$. It is easy to see that

$$
\mathcal{H}_{\infty}^{\alpha}(A)=0 \quad \text { if and only if } \quad \mathcal{H}^{\alpha}(A)=0 .
$$


Proof of Lemma 3. By linearity, it suffices to establish the lemma for $\nu \geq 0$. Let

$$
A_{k}=\left\{x \in \Omega: \nu\left(B_{r}(x)\right) \geq k r^{N-2+\beta} \text { for some } r>0\right\} .
$$

(Here, $\nu$ is viewed as a measure in $\mathbb{R}^{N}$ such that $\nu\left(\mathbb{R}^{N} \backslash \Omega\right)=0$ ).

We claim that (4.3) and (4.4) hold for $A_{k}$. We begin by establishing (4.4). For each $x \in A_{k}$, let $r_{x}>0$ be such that

$$
\nu\left(B_{r_{x}}(x)\right) \geq k r_{x}^{N-2+\beta} .
$$

Clearly, $\left(B_{5 r_{x}}(x)\right)_{x \in A_{k}}$ is a covering of $A_{k}$. Applying Vitali's covering lemma, we may extract a subcovering $\left(B_{5 r_{i}}\left(x_{i}\right)\right)$ of $A_{k}$ such that the balls $B_{r_{i}}\left(x_{i}\right)$ are all disjoint. We then have

$$
\begin{aligned}
\mathcal{H}_{\infty}^{N-2+\beta}\left(A_{k}\right) & \leq \sum_{i}\left(5 r_{i}\right)^{N-2+\beta} \\
& =C \sum_{i} r_{i}^{N-2+\beta} \\
& \leq \frac{C}{k} \sum_{i} \nu\left(B_{r_{i}}\left(x_{i}\right)\right)=\frac{C}{k} \nu\left(\bigcup_{i} B_{r_{i}}\left(x_{i}\right)\right) \leq \frac{C}{k}\|\nu\|_{\mathcal{M}} .
\end{aligned}
$$

This is precisely (4.4). We now turn to the proof of (4.3). We shall closely follow the argument presented in [8]. For simplicity, we assume $N \geq 3$; the case $N=2$ follows along the same lines.

Clearly, it suffices to prove (4.3) for the function $w$ defined as

$$
w(x)=\frac{1}{N(N-2) \omega_{N}} \int_{\Omega} \frac{d \nu(z)}{|z-x|^{N-2}} \quad \forall x \in \Omega,
$$

where $\omega_{N}=\left|B_{1}\right|$ is the measure of the unit ball in $\mathbb{R}^{N}$. It is not difficult to see that $w$ can be rewritten as (see e.g. [18, Lemma 2])

$$
w(x)=\frac{1}{N \omega_{N}} \int_{0}^{\infty} \frac{\nu\left(B_{s}(x)\right)}{s^{N-1}} d s .
$$

Given $x, y \in \Omega \backslash A_{k}$, let $\delta=|x-y|$. We then write

$$
\begin{aligned}
w(x)-w(y) & =\frac{1}{N \omega_{N}} \int_{0}^{\infty}\left[\nu\left(B_{s}(x)\right)-\nu\left(B_{s}(y)\right)\right] \frac{d s}{s^{N-1}} \\
& =\frac{1}{N \omega_{N}}\left\{\int_{0}^{2 \delta}+\int_{2 \delta}^{\infty}\right\} .
\end{aligned}
$$


Since $x, y \notin A_{k}$,

$$
\nu\left(B_{s}(x)\right), \nu\left(B_{s}(y)\right) \leq k s^{N-2+\beta} \quad \forall s>0 .
$$

We then have

$$
\int_{0}^{2 \delta} \leq \int_{0}^{2 \delta} \nu\left(B_{s}(x)\right) \frac{d s}{s^{N-1}} \leq k \int_{0}^{2 \delta} \frac{d s}{s^{1-\beta}}=C k \delta^{\beta} .
$$

On the other hand, for $s \geq 2 \delta$, we have $B_{s-\delta}(x) \subset B_{s}(y)$; thus,

$$
\begin{aligned}
\int_{2 \delta}^{\infty} & \leq \int_{2 \delta}^{\infty}\left[\nu\left(B_{s}(x)\right)-\nu\left(B_{s-\delta}(x)\right)\right] \frac{d s}{s^{N-1}} \\
& \leq \int_{\delta}^{\infty} \nu\left(B_{s}(x)\right)\left\{\frac{1}{s^{N-1}}-\frac{1}{(s+\delta)^{N-1}}\right\} d s .
\end{aligned}
$$

Since

$$
\frac{1}{s^{N-1}}-\frac{1}{(s+\delta)^{N-1}} \leq C \frac{\delta}{s^{N}} \quad \forall s \geq \delta
$$

we then get

$$
\int_{2 \delta}^{\infty} \leq C \delta \int_{\delta}^{\infty} \nu\left(B_{s}(x)\right) \frac{d s}{s^{N}} \leq C k \delta \int_{\delta}^{\infty} \frac{d s}{s^{2-\beta}} \leq C k \delta^{\beta} .
$$

It follows from (4.7)-(4.9) that

$$
w(x)-w(y) \leq C k \delta^{\beta}=C k|x-y|^{\beta} .
$$

Switching the roles between $x$ and $y$ we conclude that $w$ satisfies (4.3). Since $v-w$ is a harmonic function, $v$ also verifies (4.3). The proof of the lemma is complete.

Given a Borel set $A \subset \mathbb{R}^{N}$, let

$$
\Theta^{*}(x, A)=\limsup _{t \rightarrow 0} \frac{\left|A \cap B_{t}(x)\right|}{\left|B_{t}(x)\right|},
$$

where $|\cdot|$ denotes the Lebesgue measure in $\mathbb{R}^{N}$. This function gives the density of points of $A$ which are close to $x$. Clearly, $0 \leq \Theta^{*}(x, A) \leq 1$.

Another ingredient in the proof of Theorem 12 is the next

Lemma 4 Given a Borel set $A \subset \mathbb{R}^{N}$, let

$$
F=\left\{x \in \mathbb{R}^{N}: \Theta^{*}(x, A) \geq \frac{1}{4}\right\} .
$$


Then, for every $0 \leq \alpha \leq N$ we have

$$
\mathcal{H}_{\infty}^{\alpha}(F) \leq C \mathcal{H}_{\infty}^{\alpha}(A)
$$

for some $C>0$ depending on $N$ and $\alpha$.

Proof. If $\alpha=0$, then the conclusion is clear. We now assume $\alpha>0$. Given $\varepsilon>0$, let $\left(B_{r_{i}}\left(x_{i}\right)\right)$ be a covering of $A$ such that

$$
\sum_{i} r_{i}^{\alpha} \leq \mathcal{H}_{\infty}^{\alpha}(A)+\varepsilon
$$

Let

$$
F_{1}=F \cap\left[\bigcup_{i} B_{2 r_{i}}\left(x_{i}\right)\right] \text { and } \quad F_{2}=F \backslash\left[\bigcup_{i} B_{2 r_{i}}\left(x_{i}\right)\right]
$$

Clearly,

$$
\mathcal{H}_{\infty}^{\alpha}\left(F_{1}\right) \leq \sum_{i}\left(2 r_{i}\right)^{\alpha} \leq 2^{\alpha}\left[\mathcal{H}_{\infty}^{\alpha}(A)+\varepsilon\right] .
$$

We now prove a similar estimate for $\mathcal{H}_{\infty}^{\alpha}\left(F_{2}\right)$. Since (4.10) holds, for each $y \in F_{2}$ one can find $s_{y}>0$ sufficiently small so that

$$
\left|A \cap B_{s_{y} / 2}(y)\right| \geq \frac{1}{8}\left|B_{s_{y} / 2}(y)\right| .
$$

Applying Vitali's covering lemma to $\left(B_{5 s_{y}}(y)\right)_{y \in F_{2}}$, we may extract a subcovering $\left(B_{5 s_{j}}\left(y_{j}\right)\right)$ of $F_{2}$ such that the balls $B_{s_{j}}\left(y_{j}\right)$ are disjoint. For each $j$, we define

$$
I_{j}=\left\{i: x_{i} \in B_{s_{j}}\left(y_{j}\right)\right\} .
$$

In particular, the sets $I_{j}$ are disjoint. We claim that

$$
s_{j}^{\alpha} \leq C_{N, \alpha} \sum_{i \in I_{j}} r_{i}^{\alpha} \quad \forall j \geq 1 .
$$

In order to establish (4.15), we first observe that

$$
A \cap B_{s_{j} / 2}\left(y_{j}\right) \subset \bigcup_{i \in I_{j}} B_{r_{i}}\left(x_{i}\right) .
$$

In fact, given $z \in A \cap B_{s_{j} / 2}\left(y_{j}\right)$, let $i$ be such that $z \in B_{r_{i}}\left(x_{i}\right)$. We claim that $i \in I_{j}$. Assume by contradiction that $i \notin I_{j}$, i.e. suppose $x_{i} \notin B_{s_{j}}\left(y_{j}\right)$. Since

$$
s_{j} \leq d\left(x_{i}, y_{j}\right) \leq d\left(x_{i}, z\right)+d(z, y)<r_{i}+\frac{s_{j}}{2}
$$


we deduce that $\frac{s_{j}}{2}<r_{i}$ and then $d\left(x_{i}, y_{j}\right)<2 r_{i}$. In other words, $y_{j} \in B_{2 r_{i}}\left(x_{i}\right)$, which contradicts the definition of $F_{2}$, since $y_{j} \in F_{2}$. This establishes (4.16). Applying (4.14) and (4.16), we have

$$
\left(\frac{s_{j}}{2}\right)^{N}=\frac{1}{\omega_{N}}\left|B_{s_{j} / 2}\left(y_{j}\right)\right| \leq \frac{8}{\omega_{N}}\left|A \cap B_{s_{j} / 2}\left(y_{j}\right)\right| \leq \frac{8}{\omega_{N}} \sum_{i \in I_{j}}\left|B_{r_{i}}\left(x_{i}\right)\right|=8 \sum_{i \in I_{j}} r_{i}^{N} .
$$

Since $0<\alpha \leq N$, we conclude that (4.15) holds. It now follows from (4.15) that

$$
\mathcal{H}_{\infty}^{\alpha}\left(F_{2}\right) \leq \sum_{j=1}^{\infty}\left(5 s_{j}\right)^{\alpha} \leq 5^{\alpha} C_{N, \alpha} \sum_{j=1}^{\infty} \sum_{i \in I_{j}} r_{i}^{\alpha} \leq C \sum_{i} r_{i}^{\alpha} \leq C\left[\mathcal{H}_{\infty}^{\alpha}(A)+\varepsilon\right] .
$$

Combining (4.13) and (4.17), we obtain

$$
\mathcal{H}_{\infty}^{\alpha}(F) \leq C\left[\mathcal{H}_{\infty}^{\alpha}(A)+\varepsilon\right] .
$$

Since $\varepsilon>0$ was arbitrary, (4.11) follows.

We now present the

Proof of Theorem 12 when $0<\beta<1$. Without loss of generality, we may assume that $v=0$ on $\partial \Omega$; the general case follows by taking $v \varphi$, where $\varphi$ is any function such that $\varphi \in C_{\mathrm{c}}^{\infty}(\Omega)$ and $0 \leq \varphi \leq 1$ in $\Omega$.

Fix $k \geq 1$ and let $A_{k}$ be the set given by Lemma 3 . We have

$$
[v=1] \subset A_{k} \cup E_{k},
$$

where $E_{k}=[v=1] \backslash A_{k}$. We further decompose $E_{k}$ as

$$
E_{k}=E_{k, 1} \cup E_{k, 2},
$$

where

$$
E_{k, 1}=\left\{x \in E_{k}: \Theta^{*}\left(x, A_{k}\right) \geq \frac{1}{4}\right\} \quad \text { and } \quad E_{k, 2}=\left\{x \in E_{k}: \Theta^{*}\left(x, A_{k}\right)<\frac{1}{4}\right\} .
$$

By Lemma 4, we have

$$
\mathcal{H}_{\infty}^{N-2+\beta}\left(E_{k, 1}\right) \leq C \mathcal{H}_{\infty}^{N-2+\beta}\left(A_{k}\right) .
$$

We now claim that

$$
\limsup _{t \rightarrow 0} \frac{1}{t^{N-2+\beta}} \int_{B_{t}(x)} g(v)>0 \quad \forall x \in E_{k, 2} .
$$


In fact, given $x \in E_{k, 2}$, let $t_{0}>0$ be sufficiently small so that

$$
\left|A_{k} \cap B_{t}(x)\right| \leq \frac{1}{4}\left|B_{t}(x)\right| \quad \forall t \in\left(0, t_{0}\right) .
$$

Recall that $x \in \Omega \backslash A_{k}$ and $v(x)=1$. It follows from (4.3) that

$$
v(y) \geq 1-C k|x-y|^{\beta} \geq 1-C k t^{\beta} \quad \forall y \in B_{t}(x) \backslash A_{k} .
$$

Since (1.4) holds, there exist $\tilde{C}_{k}>0$ and a sequence $t_{n} \downarrow 0$ such that

$$
g\left(1-C k t_{n}^{\beta}\right) \geq \frac{\tilde{C}_{k}}{t_{n}^{2-\beta}} \quad \forall n \geq 1 .
$$

Thus, for every $n \geq 1$ sufficiently large, we get

$$
g(v(y)) \geq \frac{\tilde{C}_{k}}{t_{n}^{2-\beta}} \quad \forall y \in B_{t_{n}}(x) \backslash A_{k} .
$$

Since (4.20) holds, we obtain

$$
\int_{B_{t_{n}}(x)} g(v) \geq \int_{B_{t_{n}}(x) \backslash A_{k}} g(v) \geq \frac{3}{4}\left|B_{t_{n}}(x)\right| \frac{\tilde{C}_{k}}{t_{n}^{2-\beta}}=C_{k} t_{n}^{N-2+\beta},
$$

which gives (4.19). It now follows from (4.19) that $\mathcal{H}^{N-2+\beta}\left(E_{k, 2}\right)=0$ (see e.g. $[15$, p.77]); equivalently, we have

$$
\mathcal{H}_{\infty}^{N-2+\beta}\left(E_{k, 2}\right)=0 .
$$

We now deduce from (4.18) and (4.21) that

$$
\mathcal{H}_{\infty}^{N-2+\beta}\left(E_{k}\right) \leq C \mathcal{H}_{\infty}^{N-2+\beta}\left(A_{k}\right)
$$

Therefore,

$$
\mathcal{H}_{\infty}^{N-2+\beta}([v=1]) \leq C \mathcal{H}_{\infty}^{N-2+\beta}\left(A_{k}\right) \leq \frac{C}{k}\|\nu\|_{\mathcal{M}} .
$$

Since this estimate holds true for every $k \geq 1$, as we let $k \rightarrow+\infty$ we obtain

$$
\mathcal{H}_{\infty}^{N-2+\beta}([v=1])=0 .
$$

In view of (4.5), the result follows.

The proof of Theorem 12 in the case $1 \leq \beta<2$ follows the same strategy, although it is more technical. For this reason we shall indicate the main steps in the proof. The counterpart of Lemma 3 is given by the following 
Lemma 5 Let $\nu \in \mathcal{M}(\Omega)$ and let $v$ be the solution of (4.2). Given $1 \leq \beta<2$ and $k \geq 1$, there exists a Borel set $A_{k} \subset \Omega$ such that

$$
\left|2 v\left(\frac{x+y}{2}\right)-v(x)-v(y)\right| \leq C k|x-y|^{\beta}
$$

for every $x, y \in \Omega \backslash A_{k}$ such that $\frac{x+y}{2} \in \Omega \backslash A_{k}$; moreover,

$$
\mathcal{H}_{\infty}^{N-2+\beta}\left(A_{k}\right) \leq \frac{C}{k}\|\nu\|_{\mathcal{M}},
$$

for some constant $C>0$ independent of $k$.

Proof. It suffices to consider the case where $\nu \geq 0$. Let $A_{k}$ be given by (4.6). Proceeding as in the proof of Lemma 3, we obtain (4.23). We assume $N \geq 3$. We now show that $w$ defined by

$$
w(x)=a_{N} \int_{\Omega} \frac{d \nu(z)}{|z-x|^{N-2}} \quad \forall x \in \Omega,
$$

where $a_{N}=\frac{1}{N(N-2) \omega_{N}}$, satisfies property (4.22). Let $x, y \in \Omega \backslash A_{k}$ be such that $\frac{x+y}{2} \in \Omega \backslash A_{k}$. Set $\delta=|x-y|$. We have

$$
\begin{aligned}
\mid 2 w\left(\frac{x+y}{2}\right)-w(x) & -w(y) \mid \leq \\
& \leq a_{N} \int_{\Omega}\left|\frac{2}{\left|z-\frac{x+y}{2}\right|^{N-2}}-\frac{1}{|z-x|^{N-2}}-\frac{1}{|z-y|^{N-2}}\right| d \mu(z) .
\end{aligned}
$$

We split this integral into two parts:

$$
\frac{1}{a_{N}}\left|2 w\left(\frac{x+y}{2}\right)-w(x)-w(y)\right| \leq \int_{\left|z-\frac{x+y}{2}\right|<2 \delta}+\int_{\left|z-\frac{x+y}{2}\right| \geq 2 \delta} .
$$

Note that $B_{2 \delta}\left(\frac{x+y}{2}\right) \subset B_{\frac{5 \delta}{2}}(x) \cap B_{\frac{5 \delta}{2}}(y)$. Thus,

$$
\begin{aligned}
\int_{\left|z-\frac{x+y}{2}\right|<2 \delta} & \leq 2 \int_{B_{2 \delta}\left(\frac{x+y}{2}\right)} \frac{d \nu(z)}{\left|z-\frac{x+y}{2}\right|^{N-2}}+\int_{B_{\frac{5 \delta}{2}}(x)} \frac{d \nu(z)}{|z-x|^{N-2}}+\int_{B_{\frac{5 \delta}{2}}(y)} \frac{d \nu(z)}{|z-y|^{N-2}} \\
& \leq C \int_{0}^{\frac{5 \delta}{2}}\left[2 \nu\left(B_{s}\left(\frac{x+y}{2}\right)\right)+\nu\left(B_{s}(x)\right)+\nu\left(B_{s}(y)\right)\right] \frac{d s}{s^{N-1}} \\
& \leq C k \delta^{\beta} .
\end{aligned}
$$

On the other hand, we have

$$
\left|\frac{2}{\left|z-\frac{x+y}{2}\right|^{N-2}}-\frac{1}{|z-x|^{N-2}}-\frac{1}{|z-y|^{N-2}}\right| \leq C \frac{\delta^{2}}{\left|z-\frac{x+y}{2}\right|^{N}}
$$


if $\left|z-\frac{x+y}{2}\right| \geq 2 \delta$. Therefore,

$$
\begin{aligned}
\int_{\left|z-\frac{x+y}{2}\right| \geq 2 \delta} & \leq C \delta^{2} \int_{\left|z-\frac{x+y}{2}\right| \geq 2 \delta} \frac{d \nu(z)}{\left|z-\frac{x+y}{2}\right|^{N}} \\
& \leq C N \delta^{2} \int_{2 \delta}^{\infty} \nu\left(B_{s}\left(\frac{x+y}{2}\right)\right) \frac{d s}{s^{N+1}} \\
& \leq C N k \delta^{2} \int_{2 \delta}^{\infty} \frac{d s}{s^{3-\beta}} \leq C k \delta^{\beta} .
\end{aligned}
$$

As in the proof of Lemma 3, we conclude that (4.22) holds.

We now present the

Proof of Theorem 12 completed. Assume $1 \leq \beta<2$. Let $E_{k, 1}$ and $E_{k, 2}$ be defined as in the case $0<\beta<1$; in particular,

$$
[v=1] \subset A_{k} \cup E_{k, 1} \cup E_{k, 2} .
$$

By Lemma 4 we have

$$
\mathcal{H}_{\infty}^{N-2+\beta}\left(E_{k, 1}\right) \leq C \mathcal{H}_{\infty}^{N-2+\beta}\left(A_{k}\right) .
$$

In order to establish the theorem, we are left to prove (4.21). Given $x \in E_{2, k}$, let $R_{x}$ denote the reflexion with respect to $x$; namely,

$$
R_{x}(y)=2 x-y \quad \forall y \in \mathbb{R}^{N} .
$$

We claim that

$$
v(y) \geq 1-C k t^{\beta} \quad \forall y \in B_{t}(x) \backslash\left(A_{k} \cup R_{x} A_{k}\right) .
$$

In fact, for every $y \in B_{t}(x) \backslash\left(A_{k} \cup R_{x} A_{k}\right)$, we have $R_{x}(y) \in \Omega \backslash A_{k}$. Since $x \in \Omega \backslash A_{k}$, $v(x)=1$ and $v \leq 1$, we get

$$
v(y) \geq v(y)+v\left(R_{x} y\right)-1 \geq 1-C k|x-y|^{\beta} \geq 1-C k t^{\beta},
$$

which is precisely (4.24). We now take $t_{0}>0$ sufficiently small so that

$$
\left|A_{k} \cap B_{t}(x)\right| \leq \frac{1}{4}\left|B_{t}(x)\right| \quad \forall t \in\left(0, t_{0}\right) .
$$

Therefore,

$$
\left|\left(A_{k} \cup R_{x} A_{k}\right) \cap B_{t}(x)\right| \leq \frac{1}{2}\left|B_{t}(x)\right| \quad \forall t \in\left(0, t_{0}\right) .
$$


We can now proceed as in the case $0<\beta<1$ to conclude that

$$
\limsup _{t \rightarrow 0} \frac{1}{t^{N-2+\beta}} \int_{B_{t}(x)} g(v)>0 \quad \forall x \in E_{k, 2} .
$$

Thus,

$$
\mathcal{H}_{\infty}^{N-2+\beta}\left(E_{k, 2}\right)=0 .
$$

As before, we deduce that (4.1) holds. The proof of Theorem 12 is complete.

\section{Capacitary estimates related to problem (1.1)}

In this section we prove some estimates on the capacity of the set $\left[u^{*}=1\right]$. They should be compared with the result of Theorem 12 concerning the Hausdorff measure of this set. We assume throughout this section that $g$ satisfies a slightly stronger hypothesis than (1.4), namely

$$
\liminf _{t \uparrow 1}\left\{(1-t)^{\frac{2-\beta}{\beta}} g(t)\right\}>0 .
$$

Given $p>1$ and a Borel set $E \subset \Omega$, we shall denote by $\operatorname{cap}_{p}(E)$ the capacity of $E$ associated to $W_{0}^{1, p}(\Omega)$. Note that cap ${ }_{2}$ coincides with the $H^{1}$-capacity, denoted by cap elsewhere in this paper.

Our goal in this section is to establish the

Theorem 13 Let $v \in L^{1}(\Omega), v \leq 1$ a.e., be such that $\Delta v \in \mathcal{M}(\Omega)$. Assume that $g$ satisfies (5.1) for some $\beta \in(0,1]$. If $g(v) \in L^{1}(\Omega)$ then

$$
\operatorname{cap}_{2-\beta}([v=1])=0
$$

Note that $\beta \in(0,1]$ implies $2-\beta \geq 1$, so that $\operatorname{cap}_{2-\beta}$ is well-defined.

Proof.

Step 1. Proof of (5.2) if $v \in W_{0}^{1,1}(\Omega)$.

Set $\eta(s)=\frac{s^{+}}{1-s}$ and let $T_{k}(s)=\min \{s, k\}$. Since $v \in W_{0}^{1,1}(\Omega)$, for every $k \geq 1$ we have

$$
\int_{\Omega} \nabla v \cdot \nabla T_{k}(\eta(v)) d x \leq k\|\Delta v\|_{\mathcal{M}} .
$$

Indeed, inequality (5.3) (which formally amounts to multiplying $\Delta v$ by $T_{k}(\eta(v))$ ) can be obtained by approximating $v$ (e.g. through convolution) with smooth functions $v_{n}$ such that $\left\|\Delta v_{n}\right\|_{\mathcal{M}} \leq\|\Delta v\|_{\mathcal{M}}$. 
We can rewrite $(5.3)$ as

$$
\int_{[\eta(v)<k]} \frac{\left|\nabla v^{+}\right|^{2}}{(1-v)^{2}} d x \leq k\|\Delta v\|_{\mathcal{M}}
$$

On the other hand, applying Hölder's inequality with exponents $\frac{2}{2-\beta}$ and $\frac{2}{\beta}$, we have

$$
\begin{aligned}
& \int_{[\eta(v)<k]} \frac{\left|\nabla v^{+}\right|^{2-\beta}}{(1-v)^{2(2-\beta)}} d x \leq \\
& \quad \leq\left(\int_{[\eta(v)<k]} \frac{\left|\nabla v^{+}\right|^{2}}{(1-v)^{2}} d x\right)^{1-\frac{\beta}{2}}\left(\int_{[\eta(v)<k]} \frac{1}{(1-v)^{\frac{2(2-\beta)}{\beta}}} d x\right)^{\frac{\beta}{2}} .
\end{aligned}
$$

It then follows from (5.4)-(5.5) and the definition of $\eta$ that

$$
\int_{[\eta(v)<k]} \frac{\left|\nabla v^{+}\right|^{2-\beta}}{(1-v)^{2(2-\beta)}} d x \leq c\left(k\|\Delta v\|_{\mathcal{M}}\right)^{1-\frac{\beta}{2}}\left(\int_{[\eta(v)<k]} 1+\frac{\eta(v)^{\frac{2-\beta}{\beta}}}{(1-v)^{\frac{2-\beta}{\beta}}} d x\right)^{\frac{\beta}{2}} .
$$

By assumption (5.1) there exists a constant $c_{0}>0$ such that

$$
g(t)(1-t)^{\frac{2-\beta}{\beta}} \geq c_{0} \quad \forall t \in\left(\frac{1}{2}, 1\right) .
$$

From (5.6), we obtain

$$
\begin{aligned}
& \int_{[\eta(v)<k]} \frac{\left|\nabla v^{+}\right|^{2-\beta}}{(1-v)^{2(2-\beta)}} d x \leq \\
& \leq c\left(k\|\Delta v\|_{\mathcal{M})^{1-\frac{\beta}{2}}}\left(\int_{[\eta(v)<k]}\left[1+g(v) \eta(v)^{\frac{2-\beta}{\beta}}\right] d x\right)^{\frac{\beta}{2}}\right. \\
& \leq c k^{2-\beta}\|\Delta v\|_{\mathcal{M}}^{1-\frac{\beta}{2}}\left(\int_{\Omega} \frac{1+g(v) T_{k}(\eta(v))^{\frac{2-\beta}{\beta}}}{k^{\frac{2-\beta}{\beta}}} d x\right)^{\frac{\beta}{2}}
\end{aligned}
$$

Since $g(v) \in L^{1}(\Omega)$ (which also implies that $\eta(v)$ is finite a.e.) we have

$$
\lim _{k \rightarrow+\infty} \int_{\Omega} \frac{1+g(v) T_{k}(\eta(v))^{\frac{2-\beta}{\beta}}}{k^{\frac{2-\beta}{\beta}}} d x=0 .
$$

We then deduce from (5.7) that

$$
\lim _{k \rightarrow+\infty} \int_{\Omega}\left|\frac{\nabla T_{k}(\eta(v))}{k}\right|^{2-\beta} d x=0 .
$$


Note that

$$
\frac{T_{k}(\eta(v))}{k} \geq 1 \quad \text { in }[\eta(v) \geq k]
$$

Therefore,

$$
\operatorname{cap}_{2-\beta}([\eta(v) \geq k]) \leq \int_{\Omega}\left|\frac{\nabla T_{k}(\eta(v))}{k}\right|^{2-\beta} d x \stackrel{k \rightarrow+\infty}{\longrightarrow} 0 .
$$

Since

$$
[v=1]=[\eta(v)=+\infty]=\bigcap_{k=1}^{\infty}[\eta(v) \geq k]
$$

we conclude that

$$
\operatorname{cap}_{2-\beta}([v=1])=0 .
$$

Step 2. Proof of Theorem 13 completed.

We replace $v$ with $v \varphi$ where $\varphi \in C_{\mathrm{c}}^{\infty}(\Omega)$ is a cut-off function, i.e. $0 \leq \varphi \leq 1$ in $\Omega$ and $\varphi=1$ on a compact set $K \subset \Omega$. Since $v$ and $\nabla v \in L_{\text {loc }}^{1}(\Omega)$, it follows that $\Delta(v \varphi) \in \mathcal{M}(\Omega)$. Moreover $g(v \varphi) \leq g(v)$ a.e., hence $g(v \varphi) \in L^{1}(\Omega)$. We can then apply the previous step to $v \varphi$ to deduce that $\operatorname{cap}_{2-\beta}([v \varphi=1])=0$. Thus,

$$
\operatorname{cap}_{2-\beta}([v=1] \cap K)=0 \quad \text { for every compact } K \subset \Omega \text {. }
$$

By subadditivity of $\mathrm{cap}_{2-\beta}$, we conclude that

$$
\operatorname{cap}_{2-\beta}([v=1])=0 .
$$

It is well-known (see e.g. [15]) that $\operatorname{cap}_{1}(E)=0$ if and only if $\mathcal{H}^{N-1}(E)=0$. Thus, in the case $\beta=1$, we recover Theorem 12 but with a totally different proof. On the other hand, for any $p>1, \operatorname{cap}_{p}(E)=0$ implies $\mathcal{H}^{s}(E)=0$ for any $s>N-p$ (but the converse is not true). Thus, for $\beta \in(0,1)$, Theorem 13 only gives $\mathcal{H}^{s}([v=1])=0$ for any $s>N-2+\beta$, which is not optimal in view of Theorem 12 .

However, it should be noticed that the proof of Theorem 13 only relies on energy estimates, which remain true for more general operators, for instance in the inhomogeneous case. Namely, assume $A(x)=\left(a_{i, j}(x)\right)$ is an $N \times N$-matrix with bounded measurable coefficients satisfying

$$
\lambda_{1}|\xi|^{2} \leq A(x) \xi \cdot \xi \leq \lambda_{2}|\xi|^{2} \quad \forall \xi \in \mathbb{R}^{N}, \quad \text { for a.e. } x \in \Omega
$$


where $0<\lambda_{1} \leq \lambda_{2}$. Proceeding as in the proof of Theorem 13, one deduces the following result:

Theorem 14 Let $v \in L^{1}(\Omega)$ be such that $\operatorname{div}(A(x) \nabla v)$ is a bounded measure "in the sense of Stampacchia", i.e. assume there exists $\mu \in \mathcal{M}(\Omega)$ such that

$$
-\int_{\Omega} v \operatorname{div}\left(A^{*}(x) \nabla \zeta\right) d x=\int_{\Omega} \zeta d \mu
$$

for every $\zeta \in C_{0}(\bar{\Omega}) \cap H_{0}^{1}$ such that $\operatorname{div}\left(A^{*}(x) \nabla \zeta\right) \in L^{\infty}(\Omega)$. Assume $g$ satisfies (5.1) for some $\beta \in(0,1]$. If $g(v) \in L^{1}(\Omega)$, then we have

$$
\operatorname{cap}_{2-\beta}([v=1])=0 .
$$

Proof. Let $\mu_{n}$ be a suitable smooth convolution of $\mu$, and consider the solutions $v_{n}$ of

$$
\left\{\begin{array}{l}
-\operatorname{div}\left(A(x) \nabla v_{n}\right)=\mu_{n} \quad \text { in } \Omega, \\
v_{n} \in H_{0}^{1}(\Omega) .
\end{array}\right.
$$

Multiplying this equation by $T_{k}\left(\eta\left(v_{n}\right)\right)$ (see the definition of $\eta(s)$ in Step 1 of Theorem 13), we get

$$
\lambda_{1} \int_{\left[\eta\left(v_{n}\right)<k\right]} \frac{\left|\nabla v_{n}^{+}\right|^{2}}{\left(1-v_{n}\right)^{2}} d x \leq \int_{\Omega}\left(A(x) \nabla v_{n}\right) \cdot \nabla T_{k}\left(\eta\left(v_{n}\right)\right) d x \leq k\left\|\mu_{n}\right\|_{\mathcal{M}} \leq k\|\mu\|_{\mathcal{M}} .
$$

Since the solutions in the sense of Stampacchia are unique and stable, $v_{n}$ converges to $v$ in $L^{1}(\Omega)$. Therefore, as $n \rightarrow+\infty$, we obtain

$$
\lambda_{1} \int_{[\eta(v)<k]} \frac{\left|\nabla v^{+}\right|^{2}}{(1-v)^{2}} d x \leq k\|\mu\|_{\mathcal{M}}
$$

Henceforth, one can follow the proof of Step 1 of Theorem 13 in order to conclude.

In particular, if $v$ satisfies the assumptions of Theorem 14, then

$$
\mathcal{H}^{s}([v=1])=0 \quad \text { for any } s>N-2+\beta, \quad \text { if } \beta \in(0,1) .
$$

Note that

$$
\mathcal{H}^{N-1}([v=1])=0 \quad \text { if } \beta=1 .
$$

It is an open problem whether (5.10) holds with $s=N-2+\beta$, where $\beta \in(0,2)$, $\beta \neq 1$. Note that, in the inhomogeneous case, it is not clear how to implement an approach based on Hölder continuity, as used in the proof of Theorem 12. 
Remark 1 In the same spirit, the proof of Theorem 13 extends to nonlinear operators, as e.g. the $p$-Laplacian, for functions $v$ which satisfy $-\operatorname{div}\left(|\nabla v|^{p-2} \nabla v\right) \in$ $\mathcal{M}(\Omega)$ "in the renormalized sense" (see [9] for the precise definition). In this case, one can prove with the same method that if (5.1) holds true for some $\beta \in(0,1]$ and $g(v) \in L^{1}(\Omega)$, then

$$
\operatorname{cap}_{q}([v=1])=0 \quad \text { with } q=\frac{(2-\beta) p}{2(1-\beta)+\beta p} .
$$

Note that if $\beta=1$, then it still holds that

$$
\operatorname{cap}_{1}([v=1])=0=\mathcal{H}^{N-1}([v=1])
$$

\section{Every diffuse measure is good for some $g$}

Our goal in this section is to establish the following

Theorem 15 Let $\mu \in \mathcal{M}(\Omega)$ be such that $\mu^{+}$is diffuse. Then, there exists some $g$ such that $\mu \in \mathcal{G}(g)$.

We shall start with the

Proposition 5 Let $g_{1}, g_{2}$ be such that $g_{1} \leq g_{2}$. Then, $\mathcal{G}\left(g_{1}\right) \subset \mathcal{G}\left(g_{2}\right)$.

Proof. Given $\mu \in \mathcal{G}\left(g_{1}\right)$, let $u$ be the solution of

$$
\left\{\begin{aligned}
-\Delta u+g_{1}(u)=\mu & & \text { in } \Omega, \\
u=0 & & \text { on } \partial \Omega .
\end{aligned}\right.
$$

Let $\mu^{*}$ be the reduced measure relative to $g_{2}$ and denote by $u^{*}$ the solution of

$$
\left\{\begin{aligned}
-\Delta u^{*}+g_{2}\left(u^{*}\right) & =\mu^{*} & & \text { in } \Omega, \\
u^{*} & =0 & & \text { on } \partial \Omega .
\end{aligned}\right.
$$

Since $\mu^{*} \leq \mu$ and $g_{2} \geq g_{1}$, we have $u^{*} \leq u$ (see [4, Corollary B.2]). In other words, $u-u^{*} \geq 0$ in $\Omega$ and $u-u^{*}=0$ on the set $\left[u^{*}=1\right]$. Thus, by $(2.3)$ we have

$$
\left(\mu^{*}-\mu\right)_{\mathrm{d}}=\left[\Delta\left(u-u^{*}\right)\right]_{\mathrm{d}} \geq 0 \quad \text { in }\left[u^{*}=1\right] .
$$

This implies $\left(\mu^{*}\right)_{\mathrm{d}}=\mu_{\mathrm{d}}$ in $\left[u^{*}=1\right]$. On the other hand, by Theorem 4 ,

$$
\left(\mu^{*}\right)_{\mathrm{d}}=\mu_{\mathrm{d}} \quad \text { in }\left[u^{*}<1\right] .
$$


We conclude that

$$
\left(\mu^{*}\right)_{\mathrm{d}}=\mu_{\mathrm{d}}
$$

Finally, since $\mu$ is a good measure relative to $g_{1}$, we have $\mu_{\mathrm{c}} \leq 0$. Thus, by $(2.10)$,

$$
\left(\mu^{*}\right)_{\mathrm{c}}=-\left(\mu_{\mathrm{c}}\right)^{-}=\mu_{\mathrm{c}}
$$

It follows from $(6.1)$ and $(6.2)$ that $\mu=\mu^{*} \in \mathcal{G}\left(g_{2}\right)$. This concludes the proof of the proposition.

Related to the previous result, we point out the following

Open Problem. Assume $g_{1} \leq g_{2}$ and $\mathcal{G}\left(g_{1}\right)=\mathcal{G}\left(g_{2}\right)$. Is it true that $g_{1}=g_{2}$ ?

We now establish the

Lemma 6 Let $\mu \in \mathcal{M}(\Omega)$ be a nonnegative diffuse measure. Given $\varepsilon>0, s_{0} \in$ $(0,1)$, and a continuous nondecreasing function $g:(-\infty, 1) \rightarrow \mathbb{R}$ satisfying $(1.2)$ (1.3), then there exists $\tilde{g}:(-\infty, 1) \rightarrow \mathbb{R}$ with

$$
\tilde{g} \geq g \quad \text { in }(-\infty, 1), \quad \tilde{g}=g \quad \text { in }\left(-\infty, s_{0}\right],
$$

and such that

$$
\mu([v=1])<\varepsilon,
$$

where $v$ is the largest subsolution of the problem

$$
\left\{\begin{aligned}
-\Delta u+\tilde{g}(u) & =\mu & & \text { in } \Omega, \\
u & =0 & & \text { on } \partial \Omega .
\end{aligned}\right.
$$

Proof. Fix $t_{0} \in\left(s_{0}, 1\right)$. Let $g_{k}:(-\infty, 1) \rightarrow \mathbb{R}$ be any increasing sequence of continuous, nondecreasing functions, such that

$$
\begin{cases}g_{k}(t)=g(t) & \text { if } t \leq s_{0} \\ g_{k}(t) \geq k & \text { if } t \geq t_{0} \\ g_{k}(t) \geq g(t) & \forall t \in(-\infty, 1)\end{cases}
$$

For each $k \geq 1$, let $\mu_{k}$ denote the reduced measure of $\mu$ relative to $g_{k}$. We shall denote by $v_{k}$ the corresponding solution. In particular, by Proposition 1,

$$
\int_{\Omega}\left|\Delta v_{k}\right| \leq 2\left\|\mu_{k}\right\|_{\mathcal{M}} \leq 2\|\mu\|_{\mathcal{M}}
$$


and

$$
\int_{\Omega} g_{k}\left(v_{k}\right) \leq\|\mu\|_{\mathcal{M}}
$$

In view of (6.6), we have

$$
\left|\left[v_{k} \geq t_{0}\right]\right| \leq \frac{1}{k} \int_{\Omega} g_{k}\left(v_{k}\right) \leq \frac{1}{k}\|\mu\|_{\mathcal{M}} \rightarrow 0
$$

as $k \rightarrow+\infty$. On the other hand, the sequence $\left(v_{k}\right)$ is non-increasing; thus, there exists $v \in L^{1}(\Omega)$ such that $v_{k} \downarrow v$ in $L^{1}(\Omega)$. By (6.7), we have $v \leq t_{0}$ a.e. Moreover, since $0 \leq v_{k} \leq 1$ a.e., it follows from (6.5) that $\left(v_{k}\right)$ is bounded in $L^{\infty}(\Omega) \cap H_{0}^{1}(\Omega)$. We then conclude that $v_{k} \rightarrow v \mu$-a.e. in $\Omega$ (see e.g. [6, Lemma 2.1]). Therefore,

$$
\mu\left(\left[v_{k}>t_{0}\right]\right) \rightarrow 0 \quad \text { as } k \rightarrow+\infty .
$$

The lemma then follows by taking $\tilde{g}=g_{k_{0}}$ for some $k_{0} \geq 1$ sufficiently large.

We now present the

Proof of Theorem 15. We shall split the proof of the theorem into two steps.

Step 1. Given $\mu \in \mathcal{M}(\Omega)$ diffuse and nonnegative, there exists $g$ satisfying (1.2)(1.3) such that $\mu \in \mathcal{G}(g)$.

We begin by constructing a sequence $\left(g_{k}\right)$ as follows. Let $g_{0}(t)=\frac{t}{1-t}, \forall t \in$ $[0,1)$. Given $g_{k}$, we apply Lemma 6 to $g=g_{k}, \varepsilon=\frac{1}{2^{k}}$ and $s_{0}=1-\frac{1}{2^{k}}$. Set $g_{k+1}=\tilde{g}$, where $\tilde{g}$ is the function given by Lemma 6 . In particular, the sequence $\left(g_{k}\right)$ is nondecreasing and

$$
g_{k}=g_{k_{0}} \quad \text { in }\left(-\infty, 1-\frac{1}{2^{k_{0}}}\right] \quad \forall k \geq k_{0} .
$$

Set

$$
g(t)=\lim _{k \rightarrow+\infty} g_{k}(t) \quad \forall t \in(-\infty, 1) .
$$

We claim that $\mu \in \mathcal{G}(g)$. In fact, let $\mu_{k}$ denote the reduced measure of $\mu$ relative to $g_{k}$. In particular, $\mu_{k}$ is also a diffuse measure. Since $g \geq g_{k}$, it follows from Proposition 5 that $\mu_{k} \in \mathcal{G}(g)$ for every $k \geq 1$. Let $v_{k}$ be the solution of

$$
\left\{\begin{aligned}
-\Delta v_{k}+g_{k}\left(v_{k}\right) & =\mu_{k} & & \text { in } \Omega, \\
v_{k} & =0 & & \text { on } \partial \Omega .
\end{aligned}\right.
$$

By Theorem 4 and the choice of $g_{k}$, we have

$$
\left\|\mu-\mu_{k}\right\|_{\mathcal{M}} \leq \mu\left(\left[v_{k}=1\right]\right) \leq \frac{1}{2^{k}} .
$$


Thus,

$$
\mu_{k} \rightarrow \mu \text { strongly in } \mathcal{M}(\Omega) .
$$

Since $\mathcal{G}(g)$ is closed, we conclude that $\mu \in \mathcal{G}(g)$ as claimed.

Step 2. Proof of the theorem completed.

Let $\mu \in \mathcal{M}(\Omega)$ be such that $\mu_{\mathrm{c}} \leq 0$; in other words, $\mu^{+}$is diffuse. We can then apply the previous step to $\mu^{+}$to conclude that there exists $g$ such that $\mu^{+} \in \mathcal{G}(g)$. Since $\mu \leq \mu^{+}$, by Proposition 4 we deduce that $\mu$ is also good for $g$.

\section{$7 \quad$ Measures which are good for every $g$}

In this section we characterize the set of measures which are always good. In order to do so, we first need to recall some notions about obstacle problems with measure data. Throughout this section, we denote by $\beta$ any maximal monotone graph (m.m.g.) of the form

$$
\beta(t)= \begin{cases}b(t) & \text { if } t<1 \\ {[b(1), \infty)} & \text { if } t=1 \\ \emptyset & \text { if } t>1\end{cases}
$$

where $b:(-\infty, 1] \rightarrow \mathbb{R}$ is a nondecreasing continuous function such that $b(t)=0$ if $t \leq 0$. Given a bounded measure $\mu$ in $\Omega$, we say that $w$ is a solution of

$$
\left\{\begin{aligned}
-\Delta w+\beta(w) \ni \mu & \text { in } \Omega, \\
w=0 & \text { on } \partial \Omega,
\end{aligned}\right.
$$

if $w \in L^{1}(\Omega), w \leq 1$ a.e., $\Delta w \in \mathcal{M}(\Omega)$, and there exists a nonnegative diffuse measure $\nu \in \mathcal{M}(\Omega)$ such that $\nu_{\mathrm{a}} \in \beta(w)$ a.e., $\nu_{\mathrm{s}}$ is concentrated on the set $[w=1]$, and

$$
-\int_{\Omega} w \Delta \zeta+\int_{\Omega} \zeta d \nu=\int_{\Omega} \zeta d \mu \quad \forall \zeta \in C_{0}^{2}(\bar{\Omega}) .
$$

(Here, $\nu_{\mathrm{a}}$ and $\nu_{\mathrm{s}}$ denote the absolutely continuous and the singular parts of $\nu$ with respect to the Lebesgue measure in $\mathbb{R}^{N}$ ).

In particular, the measure $\mu+\Delta w$ is diffuse and

$$
\mu+\Delta w=\nu \geq \inf \beta(1) \quad \text { in }[w=1] .
$$

Problem (7.2) has been studied by Dall'Aglio-Leone [11], Dall'Aglio-Dal Maso [10], Brezis-Ponce [6]; see also the references therein. It turns out that (7.2) has 
a solution if and only if $\mu^{+}$is diffuse; moreover, this solution is unique and is the largest solution of the problem

$$
\left\{\begin{aligned}
-\Delta v+b(v) \leq \mu & & \text { in } \Omega, \\
v \leq 1 & & \text { in } \Omega, \\
v=0 & & \text { on } \partial \Omega .
\end{aligned}\right.
$$

Our goal in this section is to establish the following

Theorem 16 Let $\mu \in \mathcal{M}(\Omega)$. Then, $\mu$ is good for every $g$ if and only if $\mu^{+}$is diffuse and

$$
\mu+\Delta w_{0} \in L^{1}(\Omega),
$$

where $w_{0}$ is the unique solution of the obstacle problem

$$
\left\{\begin{aligned}
-\Delta w_{0}+\beta_{0}\left(w_{0}\right) \ni \mu & \text { in } \Omega, \\
w_{0}=0 & \text { on } \partial \Omega,
\end{aligned}\right.
$$

with $\beta_{0}(s)=0$ if $s<1$ and $\beta_{0}(1)=[0, \infty)$.

Remark 2 It is known from [2] that if $\mu \in L^{1}(\Omega)$, then problem (1.1) has a solution for every $g$. This is consistent with Theorem 16 . Indeed, let $w_{0}$ be the solution of (7.6) with $\mu \in L^{1}(\Omega)$. Then, in view of (2.3), we have

$$
\mu+\Delta w_{0} \leq \mu \quad \text { on }\left[w_{0}=1\right] .
$$

Since $\mu+\Delta w_{0}$ is a nonnegative measure and it is concentrated on $\left[w_{0}=1\right]$, we conclude that

$$
0 \leq \mu+\Delta w_{0} \leq \mu \quad \text { in } \Omega
$$

Hence, $\mu \in L^{1}(\Omega)$ implies that $\mu+\Delta w_{0} \in L^{1}(\Omega)$.

In the proof of Theorem 16 we shall need the next two lemmas:

Lemma 7 Let $\beta$ be a m.m.g. and let $\mu \in \mathcal{M}(\Omega)$ be such that $\mu^{+}$is diffuse. If

$$
\mu_{\mathrm{a}}^{+} \in L^{\infty}(\Omega) \quad \text { and } \quad\left\|\mu_{\mathrm{a}}^{+}\right\|_{L^{\infty}}<\inf \beta(1),
$$

then

$$
|[w=1]|=0,
$$

where $w$ is the solution of (7.2). 
Proof. By $(2.3)$, we have $(\Delta w)_{\mathrm{d}} \leq 0$ on the set $[w=1]$. Thus,

$$
\mu_{\mathrm{d}} \geq(\mu+\Delta w)_{\mathrm{d}}=\mu+\Delta w \geq \inf \beta(1) \quad \text { in }[w=1] .
$$

Comparing the absolutely continuous part of both sides, we get

$$
\mu_{\mathrm{a}}=\left(\mu_{\mathrm{d}}\right)_{\mathrm{a}} \geq \inf \beta(1) \quad \text { a.e. in }[w=1] .
$$

In view of (7.7), we deduce that (7.8) holds.

Lemma 8 Let $\mu \in \mathcal{M}(\Omega)$ be such that $\mu^{+}$is diffuse. Given two m.m.g. $\beta_{1}, \beta_{2}$, let $w_{i}$ be the solution of (7.2) associated to $\beta_{i}, i=1,2$. If $\beta_{1} \geq \beta_{2}$, then

$$
0 \leq \mu+\Delta w_{1} \leq \mu+\Delta w_{2} \quad \text { in }\left[w_{1}=1\right] .
$$

Proof. By comparison, we have $w_{2}-w_{1} \geq 0$ a.e. In particular, $w_{2}-w_{1}=0$ in $\left[w_{1}=1\right]$. Applying (2.3), we get

$$
\left[\Delta\left(w_{2}-w_{1}\right)\right]_{\mathrm{d}} \geq 0 \quad \text { in }\left[w_{1}=1\right] .
$$

Thus, on the set $\left[w_{1}=1\right]$, we have

$$
\mu+\Delta w_{1}=\left(\mu+\Delta w_{1}\right)_{\mathrm{d}} \leq\left(\mu+\Delta w_{2}\right)_{\mathrm{d}}=\mu+\Delta w_{2} .
$$

Since $w_{1}$ is the solution of (7.2) with $\beta=\beta_{1}$, we have $\nu_{1}=\mu+\Delta w_{1} \geq 0$ in $\Omega$. We conclude that (7.9) holds.

\section{Proof of Theorem 16.}

Proof of $(\Leftarrow)$. We shall establish a slightly more general result:

Proposition 6 Let $\mu \in \mathcal{M}(\Omega)$ be such that $\mu^{+}$is diffuse. Assume that

$$
\mu+\Delta w \in L^{1}(\Omega),
$$

where $w$ is the unique solution of (7.2). Then, $\mu$ is good for every $g$ such that $g \geq \beta$.

Proof. We first assume $\mu_{\mathrm{a}}^{+} \in L^{\infty}(\Omega)$. Let $\beta_{1}$ be a m.m.g. such that

$$
\beta \leq \beta_{1} \leq g \text { and }\left\|\mu_{\mathrm{a}}^{+}\right\|_{L^{\infty}}<\inf \beta_{1}(1) .
$$


Let $w_{1}$ be the solution of (7.2) with obstacle $\beta_{1}$. We claim that

$$
\mu+\Delta w_{1} \in L^{1}(\Omega)
$$

In fact, since $w_{1}$ is the solution of an obstacle problem, the measure $\left(\mu+\Delta w_{1}\right)_{\mathrm{s}}$ is concentrated on the set $\left[w_{1}=1\right]$. By Lemma 8 above, we have

$$
0 \leq\left(\mu+\Delta w_{1}\right)_{\mathrm{s}} \leq(\mu+\Delta w)_{\mathrm{s}}=0 \text { in }\left[w_{1}=1\right] .
$$

This establishes (7.11).

On the other hand, it follows from Lemma 7 that the set $\left[w_{1}=1\right]$ has zero Lebesgue measure. We conclude that

$$
\mu+\Delta w_{1}=b_{1}\left(w_{1}\right) \quad \text { a.e. }
$$

In other words, $w_{1}$ verifies

$$
\left\{\begin{aligned}
-\Delta w_{1}+b_{1}\left(w_{1}\right)=\mu & & \text { in } \Omega, \\
w_{1}=0 & & \text { on } \partial \Omega .
\end{aligned}\right.
$$

Since $\left|\left[w_{1}=1\right]\right|=0$, by a variant of the De La Vallée-Poussin theorem (see [12, Remark 23] or [13, Theorem II.22]), one can find $g_{1}$ satisfying (1.2) such that

$$
b_{1} \leq g_{1} \leq g \quad \text { and } \quad g_{1}\left(w_{1}\right) \in L^{1}(\Omega) .
$$

Thus, in view of Proposition $4, \mu$ is a good measure for $g_{1}$. By Proposition 5, we deduce that $\mu$ is also good for $g$. Since $g \geq \beta$ was arbitrary, the result follows when $\mu_{\mathrm{a}}^{+} \in L^{\infty}(\Omega)$.

In order to establish the proposition for any measure $\mu$ satisfying (7.10), we let

$$
\mu_{n}=\min \left\{\mu_{\mathrm{a}}, n\right\}+\mu_{s} \quad \forall n \geq 1 .
$$

Proceeding as in the proof of (7.11), for every $n \geq 1$ we have

$$
\mu_{n}+\Delta w_{n} \in L^{1}(\Omega)
$$

where $w_{n}$ is the solution of (7.2) with data $\mu_{n}$. Moreover, $\left(\mu_{n}\right)_{\mathrm{a}}^{+} \in L^{\infty}(\Omega)$. Thus, $\mu_{n}$ is good for every $g \geq \beta$. As $n \rightarrow+\infty$, we deduce that $\mu$ is also good for any such $g$.

Proof of $(\Rightarrow)$. We shall need the following 
Lemma 9 Let $\mu \in \mathcal{M}(\Omega)$. Assume that $\mu$ is good for every $g$,

$$
\mu_{\mathrm{a}}^{+} \in L^{\infty}(\Omega) \quad \text { and } \quad\left\|\mu_{\mathrm{a}}^{+}\right\|_{L^{\infty}} \leq \inf \beta(1) .
$$

Let $w$ be the solution of (7.2). Then,

$$
\left\{\begin{aligned}
-\Delta w+b(w) & =\mu & & \text { in } \Omega, \\
w & =0 & & \text { on } \partial \Omega .
\end{aligned}\right.
$$

Proof. By making a small perturbation of $\mu$, it suffices to establish the result when

$$
\left\|\mu_{\mathrm{a}}^{+}\right\|_{L^{\infty}}<\inf \beta(1) .
$$

By Lemma 7 above, we know that $|[w=1]|=0$. Thus, one can find a continuous nondecreasing function $H:(-\infty, 1) \rightarrow \mathbb{R}, H \geq b$, satisfying (1.2) and such that $H(w) \in L^{1}(\Omega)$. We now take a sequence of functions $\left(g_{n}\right)$ such that

$$
g_{n} \leq H \quad \forall n \geq 1 \quad \text { and } \quad g_{n} \downarrow b \quad \text { as } n \uparrow+\infty .
$$

Since $\mu$ is good for every $g_{n}$, there exists $v_{n}$ satisfying (1.1) with nonlinearity $g_{n}$. Clearly, $v_{n} \uparrow v$, where $v \in L^{1}(\Omega)$ and $v \leq 1$ a.e. By Fatou, $v$ verifies (7.5); in particular, $v \leq w$ a.e. Thus,

$$
|[v=1]| \leq|[w=1]|=0 .
$$

On the other hand, note that

$$
g_{n}\left(v_{n}\right) \rightarrow b(v) \quad \text { a.e. on }[v<1] .
$$

Thus, by (7.15)-(7.16), we have

$$
g_{n}\left(v_{n}\right) \rightarrow b(v) \text { a.e. }
$$

Since $g_{n}\left(v_{n}\right) \leq H(w)$ a.e., it follows by dominated convergence that

$$
g_{n}\left(v_{n}\right) \rightarrow b(v) \quad \text { in } L^{1}(\Omega) .
$$

We deduce that $v$ satisfies (7.13). In particular, $v$ is also a solution of (7.2). By uniqueness, we conclude that $v=w$. This establishes the lemma.

We can now conclude the proof of Theorem 16 .

Let $\mu$ be a measure such that $\mu$ is good for every $g$. We assume in addition that $\mu_{\mathrm{a}}^{+} \in L^{\infty}(\Omega)$. Let $b_{n}:(-\infty, 1] \rightarrow \mathbb{R}$ be a sequence of nondecreasing continuous 
functions such that $b_{n}(t)=0$ if $t \leq 0, b_{n}(1)=\left\|\mu_{\mathrm{a}}^{+}\right\|_{L^{\infty}}$ and $b_{n}(t) \downarrow 0$ uniformly away from $t=1$. By Lemma 9, equation (7.13) has a solution $v_{n} \leq 1$ associated to $b_{n}$. Note that $v_{n} \uparrow v$, where $v \in L^{1}(\Omega), v \leq 1$ a.e. Moreover, passing to a subsequence if necessary, we have

$$
b_{n}\left(v_{n}\right) \rightarrow f \quad \text { weakly in } L^{\infty}(\Omega)
$$

for some $f \in L^{\infty}(\Omega)$ with $\|f\|_{L^{\infty}} \leq\left\|\mu_{\mathrm{a}}^{+}\right\|_{L^{\infty}}$.

We claim that $v=w_{0}$ a.e. In fact, note that $v$ satisfies

$$
\left\{\begin{aligned}
-\Delta v & =\mu-f & & \text { in } \Omega, \\
v & =0 & & \text { on } \partial \Omega .
\end{aligned}\right.
$$

Since

$$
0 \leq b_{n}\left(v_{n}\right) \leq b_{n}(v) \text { a.e. } \forall n \geq 1,
$$

as $n \rightarrow+\infty$ we obtain

$$
0 \leq f \leq \alpha \chi_{[v=1]} \quad \text { a.e. }
$$

where $\alpha=\left\|\mu_{\mathrm{a}}^{+}\right\|_{L^{\infty}}$. This implies that $f$ is nonnegative and concentrated on the set $[v=1]$. Therefore, $v$ verifies problem (7.6) and so $v=w_{0}$ as claimed. We conclude that

$$
\mu+\Delta w_{0}=f \in L^{\infty}(\Omega) .
$$

This establishes the theorem under the additional assumption that $\mu_{\mathrm{a}}^{+} \in L^{\infty}(\Omega)$. The general case easily follows by using an approximation argument.

Before proving Theorem 3, we start with the following

Proposition 7 Given $\mu \in \mathcal{M}(\Omega)$, let $v$ be the unique solution of

$$
\left\{\begin{aligned}
-\Delta v=\mu & \text { in } \Omega, \\
v=0 & \text { on } \partial \Omega .
\end{aligned}\right.
$$

If $v \leq 1$ a.e., then $\mu$ is good for every $g$.

Proof. Let $\alpha<1$. Since $v \leq 1$ a.e., $\alpha v$ is a supersolution of problem (1.1) with data $\alpha \mu$. Thus, by Proposition $4, \alpha \mu$ is good for every $\alpha<1$. Since $\mathcal{G}(g)$ is closed with respect to the strong topology in $\mathcal{M}(\Omega)$, we deduce that $\mu \in \mathcal{G}(g)$ for every $g$.

We now present the 
Proof of Theorem 3. The implication " $\Leftarrow$ " already follows from Proposition 7 above. We now establish the reverse implication. Let $\mu$ be a singular measure which is good for every $g$. In particular, $\mu^{+}$is diffuse. Let $\nu=\mu+\Delta w_{0}$, where $w_{0}$ is the solution of (7.6). In view of the definition of $\beta_{0}, \nu$ is a nonnegative diffuse measure concentrated on the set $\left[w_{0}=1\right]$. By $(2.3)$, on this set we have $\left(\Delta w_{0}\right)_{\mathrm{d}} \leq 0$, so that

$$
0 \leq \nu \leq \mu \text {. }
$$

On the other hand, by Theorem 16, we know that $\nu \in L^{1}(\Omega)$. Since $\mu$ is singular, we conclude that $\nu=0$, hence $w_{0}$ coincides with the unique solution $v$ of (7.17). Since $w_{0} \leq 1$ a.e., the result follows.

\section{How to construct diffuse measures which are not good}

Our goal in this section is to establish Theorem 1. The main ingredient is the following

Lemma 10 Given g, there exists $v \in C_{0}(\bar{\Omega})$ such that

$$
\Delta v \in L^{1}(\Omega), \quad v \leq 1 \text { in } \Omega, \quad \operatorname{cap}([v=1])>0 \quad \text { and } \quad g(v) \in L^{1}(\Omega) .
$$

Proof. Let $\left(\ell_{k}\right)$ be a decreasing sequence of positive numbers such that

$$
\ell_{k} \leq \theta \ell_{k-1} \quad \forall k \geq 2
$$

for some $\theta \in\left(0, \frac{1}{2}\right)$. Let $\left(k_{j}\right)$ be an increasing sequence of nonnegative integers. Both sequences $\left(\ell_{k}\right)$ and $\left(k_{j}\right)$ will be explicitly chosen later on.

We now briefly recall the construction presented in [18] of the Cantor set $F$ associated to the subsequence $\left(\ell_{k_{j}}\right)$. We shall assume for simplicity that $\Omega=Q_{1}$, the unit cube centered at 0 .

We first define a decreasing sequence of sets $\left(F_{j}\right)_{j \geq 0}$ as follows. Let $F_{0}=Q_{1}$, $k_{0}=0$ and $\ell_{0}=1$. We now proceed by induction. Assume $F_{j-1}, j \geq 1$, is the union of $2^{N k_{j-1}}$ disjoint cubes of length $\ell_{k_{j-1}}$. Let $Q_{i}$ be any component of $F_{j-1}$, and let $\tilde{Q}_{i} \subset Q_{i}$ be a smaller cube concentric to $Q_{i}$ (so that the ratio between their lengths is $\left.\frac{1}{2}+\theta \in\left(\frac{1}{2}, 1\right)\right)$. Inside $\tilde{Q}_{i}$, we select $2^{N\left(k_{j}-k_{j-1}\right)}$ cubes $Q_{i, s}$ of length $\ell_{k_{j}}$, uniformly distributed in $\tilde{Q}_{i}$. Set

$$
F_{j}=\bigcup_{i, s} Q_{i, s} .
$$


Thus, $F_{j} \subset F_{j-1}$ and $F_{j}$ is the union of $2^{N k_{j}}$ disjoint cubes of length $\ell_{k_{j}}$. The Cantor set associated to the subsequence $\left(\ell_{k_{j}}\right)$ is then defined as

$$
F=\bigcap_{j=1}^{\infty} F_{j} .
$$

We now split the proof of the lemma into two cases, whether $N \geq 3$ or $N=2$ :

Case 1. $N \geq 3$

We start with the following

Claim 1. For every $j \geq 1$, we have

$$
\operatorname{cap}\left(F_{j}, F_{j-1}\right) \leq C_{\theta} 2^{N k_{j}} \ell_{k_{j}}^{N-2},
$$

where cap $\left(F_{j}, F_{j-1}\right)$ denotes the $H^{1}$-capacity of the set $F_{j}$ with respect to $F_{j-1}$.

Since $F_{j-1}$ has $2^{N k_{j-1}}$ connected components, it suffices to show that

$$
\operatorname{cap}\left(F_{j} \cap Q_{i}, Q_{i}\right) \leq C_{\theta} 2^{N\left(k_{j}-k_{j-1}\right)} \ell_{k_{j}}^{N-2},
$$

where $Q_{i}$ is any component of $F_{j-1}$. Note that each component $Q_{i, s}$ of $F_{j} \cap Q_{i}$ has length $\ell_{k_{j}}$ and (see [18])

$$
d\left(Q_{i, s}, \partial Q_{i}\right) \geq \frac{1-2 \theta}{4} \operatorname{diam} Q_{i} .
$$

Hence,

$$
\operatorname{cap}\left(Q_{i, s}, Q_{i}\right) \leq C_{\theta} \ell_{k_{j}}^{N-2} .
$$

Recall that $Q_{i}$ contains $2^{N\left(k_{j}-k_{j-1}\right)}$ components $Q_{i, s}$. By the subadditivity of the capacity, we conclude that (8.4) holds. This concludes the proof of the claim.

By (8.3) and Theorem E.1 in [4], there exists $v_{j} \in C_{\mathrm{c}}^{\infty}\left(F_{j-1}\right)$ such that $0 \leq$ $v_{j} \leq 1$ in $\Omega, v_{j}=1$ on $F_{j}$, and

$$
\int_{\Omega}\left|\Delta v_{j}\right| \leq C 2^{N k_{j}} \ell_{k_{j}}^{N-2}
$$

Our aim is to construct the function $v$ of the form

$$
v=\sum_{j=1}^{\infty} \alpha_{j} v_{j}
$$

where $\left(\alpha_{j}\right)$ is a sequence of positive numbers to be chosen later on such that

$$
\sum_{j=1}^{\infty} \alpha_{j}=1
$$


Clearly, $v \in C_{0}(\bar{\Omega})$ and $v \leq 1$ in $\Omega$. Moreover, $v=1$ precisely on $\bigcap_{j} F_{j}=F$.

We claim that one can choose $\left(\ell_{k}\right),\left(k_{j}\right)$ and $\left(\alpha_{j}\right)$ such that

$$
\begin{aligned}
& \sum_{j=1}^{\infty} \frac{1}{2^{N k_{j}} \ell_{k_{j}}^{N-2}}<\infty \\
& \sum_{j=1}^{\infty} \alpha_{j} 2^{N k_{j}} \ell_{k_{j}}^{N-2}<\infty \\
& \sum_{j=1}^{\infty} g\left(\sum_{i=1}^{j} \alpha_{i}\right) 2^{N k_{j}} \ell_{k_{j}}^{N}<\infty
\end{aligned}
$$

In fact, let

$$
\alpha_{j}=3 \cdot 2^{-2 j} \quad \forall j \geq 1,
$$

so that (8.8) holds. Let $\left(k_{j}\right)$ be any increasing sequence of positive numbers such that

$$
\frac{g\left(1-2^{-2 j}\right)}{2^{\frac{N}{N-2}} k_{j}} \leq \frac{1}{2^{j}} \quad \forall j \geq 1 .
$$

Finally, we take $\left(\ell_{k}\right)$ satisfying (8.2) (with, say, $\theta=\frac{3}{4}$ ) and

$$
2^{N k_{j}} \ell_{k_{j}}^{N-2}=2^{j} \quad \forall j \geq 1 .
$$

It immediately follows that (8.9) and (8.10) hold. After some straightforward computation, the left-hand side of (8.11) can be estimated by

$$
\sum_{j=1}^{\infty} g\left(1-2^{-2 j}\right)\left(\frac{2^{j}}{2^{2 k_{j}}}\right)^{\frac{N}{N-2}} \leq \sum_{j=1}^{\infty} \frac{g\left(1-2^{-2 j}\right)}{2^{\frac{N}{N-2} k_{j}}},
$$

which is finite in view of our choice of $\left(k_{j}\right)$. We conclude that (8.9)-(8.11) hold. By construction, $[v=1$ ] coincides with $F$. On the other hand, by [18], we know that $\operatorname{cap}(F)>0$ if and only if

$$
\sum_{j=1}^{\infty} \frac{1}{2^{N k_{j}} \ell_{k_{j}}^{N-2}}<\infty
$$

In view of $(8.9)$, we deduce that cap $(F)>0$. Thus,

$$
\operatorname{cap}([v=1])>0 .
$$

By (8.6), (8.7) and (8.10), we have

$$
\Delta v \in L^{1}(\Omega) .
$$


Finally, it follows from (8.11) that

$$
g(v) \in L^{1}(\Omega) .
$$

The proof of the lemma is complete when $N \geq 3$.

Case 2. $N=2$.

As in the previous case, we start with the

Claim 2. For every $j \geq 1$, we have

$$
\operatorname{cap}\left(F_{j}, F_{j-1}\right) \leq C_{\theta} 4^{k_{j}}\left(\log \frac{1}{\ell_{k_{j}}}\right)^{-1} .
$$

The argument is similar to the proof of Claim 1. It suffices to observe that the analog of $(8.5)$ is

$$
\operatorname{cap}\left(Q_{i, s}, Q_{i}\right) \leq C_{\theta}\left(\log \frac{1}{\ell_{k_{j}}}\right)^{-1} .
$$

We now conclude the proof of the lemma. Let $\left(\alpha_{j}\right)$ be defined as before. Take an increasing sequence of positive integers $\left(k_{j}\right)$ such that

$$
\frac{g\left(1-2^{-2 j}\right)}{4^{4^{k}}} \leq \frac{1}{2^{j}} \quad \forall j \geq 1 .
$$

Finally, let $\left(\ell_{k}\right)$ satisfying $(8.2)$ and

$$
\ell_{k_{j}}=4^{-4^{k_{j} \cdot 2^{-j}}} \quad \forall j \geq 1 .
$$

With such choices, one can easily check that

$$
\begin{aligned}
& \sum_{j=1}^{\infty} \frac{1}{4^{k_{j}}} \log \frac{1}{\ell_{k_{j}}}<\infty \\
& \sum_{j=1}^{\infty} \alpha_{j} 4^{k_{j}}\left(\log \frac{1}{\ell_{k_{j}}}\right)^{-1}<\infty \\
& \sum_{j=1}^{\infty} g\left(\sum_{i=1}^{j} \alpha_{i}\right) 4^{k_{j}} \ell_{k_{j}}^{2}<\infty
\end{aligned}
$$

Let $v$ be given by (8.7), where $v_{j} \in C_{\mathrm{c}}^{\infty}\left(F_{j-1}\right)$ is such that $0 \leq v_{j} \leq 1$ in $\Omega, v_{j}=1$ on $F_{j}$, and

$$
\int_{\Omega}\left|\Delta v_{j}\right| \leq C 4^{k_{j}}\left(\log \frac{1}{\ell_{k_{j}}}\right)^{-1} .
$$


(The existence of such $v_{j}$ follows from Claim 2 above.) In particular, $[v=1]=F$. By (8.18), we have (see [18, Lemma 4])

$$
\operatorname{cap}([v=1])>0 .
$$

Moreover, proceeding as before, we deduce from (8.19) and (8.20) that

$$
\Delta v \in L^{1}(\Omega) \quad \text { and } \quad g(v) \in L^{1}(\Omega) .
$$

This concludes the proof of the lemma.

Using Lemma 10, we establish the following

Proposition 8 For every $g$, there exist a nonnegative function $h_{0} \in L^{1}(\Omega)$ and a compact set $K_{0} \subset \Omega$, with $\left|K_{0}\right|=0$ and cap $\left(K_{0}\right)>0$, such that for any measure $\sigma \geq 0$ supported in $K_{0}$ we have

$$
\left(h_{0}+\sigma\right)^{*}=h_{0},
$$

where $\left(h_{0}+\sigma\right)^{*}$ is the reduced measure associated to $h_{0}+\sigma$.

Proof. Take $K_{0}=[v=1]$ and $f_{0}=-\Delta v+g(v)$, where $v$ is the function constructed in Lemma 10. We begin with the following

Claim. If $\lambda$ is a good measure $\geq f_{0}$, then $\lambda\left(K_{0}\right)=0$.

We first observe that $\lambda$ is a diffuse measure. In fact, since $\lambda$ is good, we have $\lambda_{\mathrm{c}} \leq 0$ by Corollary 2. On the other hand, $\lambda \geq f_{0}$ implies $\lambda_{\mathrm{c}} \geq 0$. Thus, $\lambda_{\mathrm{c}}=0$, so that $\lambda$ is diffuse. Let $u$ be the solution of

$$
\left\{\begin{aligned}
-\Delta u+g(u)=\lambda & & \text { in } \Omega, \\
u=0 & & \text { on } \partial \Omega .
\end{aligned}\right.
$$

Clearly, $u \geq v$ a.e. Moreover, since $v=1$ in $K_{0}$, we have $u-v=0$ in $K_{0}$. Thus, by $(2.3)$,

$$
f_{0}-\lambda=\Delta(u-v)=[\Delta(u-v)]_{\mathrm{d}} \geq 0 \quad \text { in } K_{0} .
$$

In other words, $\lambda \leq f_{0}$ in $K_{0}$; thus, $\lambda=f_{0}$ in $K_{0}$. Since $\left|K_{0}\right|=0$, we deduce that $\lambda\left(K_{0}\right)=0$. This establishes the claim.

Let $h_{0}=f_{0}^{+}$. We now show that $h_{0}$ and $K_{0}$ satisfy the desired properties. In fact, let $\sigma \geq 0$ be a measure concentrated on $K_{0}$. Since $h_{0}+\sigma \geq 0$, it follows from Corollary 3 that

$$
0 \leq\left(h_{0}+\sigma\right)^{*} \leq h_{0}+\sigma \quad \text { in } \Omega .
$$


Moreover, by Corollary 1 ,

$$
\left[\left(h_{0}+\sigma\right)^{*}\right]_{\mathrm{a}}=h_{0} \quad \text { a.e. in } \Omega .
$$

Thus,

$$
f_{0} \leq h_{0} \leq\left(h_{0}+\sigma\right)^{*} \leq h_{0}+\sigma \text { in } \Omega .
$$

In particular, $\left[\left(h_{0}+\sigma\right)^{*}\right]_{\mathrm{s}}$ is concentrated on $K_{0}$. By our previous claim, we have $\left[\left(h_{0}+\sigma\right)^{*}\right]_{\mathrm{s}}=0$ in $K_{0}$. Therefore,

$$
\left(h_{0}+\sigma\right)^{*}=\left[\left(h_{0}+\sigma\right)^{*}\right]_{\mathrm{a}}=h_{0} \quad \text { in } \Omega .
$$

Remark 3 A slight modification in the construction of $v$, given by Lemma 10, allows to obtain the following further property in the statement of Proposition 8: given any $\varepsilon>0$ and any ball $B_{r} \subset \subset \Omega$, one can choose $h_{0}$ and $K_{0}$ such that

$$
\left\|h_{0}\right\|_{L^{1}}<\varepsilon \text { and } \quad K_{0} \subset B_{r} .
$$

Theorem 1 is now a consequence of Proposition 8:

Proof of Theorem 1. Given $g$, let $h_{0}, K_{0}$ be as in the statement of Proposition 8. Since cap $\left(K_{0}\right)>0$, there exists a diffuse measure $\sigma \geq 0$ concentrated on $K_{0}$ such that $\sigma\left(K_{0}\right)=1$ (see e.g. [8]). Let $\mu=h_{0}+\sigma$. By Proposition 8, we have $\mu \neq \mu^{*}$. Thus, $\mu \notin \mathcal{G}(g)$.

A slightly stronger version of Theorem 1 is the following

Theorem 17 Given g, let $h_{0} \in L^{1}(\Omega)$ and $K_{0} \subset \Omega$ be given by Proposition 8. Let $\sigma$ be a nonnegative diffuse measure supported in $K_{0}$. If $\sigma$ is good, then

$$
\|\sigma\|_{\mathcal{M}}<\left\|h_{0}\right\|_{L^{1}}
$$

Proof. Assume $\sigma$ is good. By Proposition 8, we have $\left(h_{0}+\sigma\right)^{*}=h_{0}$. Recall that, by Theorem $5,\left(h_{0}+\sigma\right)^{*}$ is the closest good measure to $h_{0}+\sigma$. Thus,

$$
\|\sigma\|_{\mathcal{M}}=\left\|\left(h_{0}+\sigma\right)-\left(h_{0}+\sigma\right)^{*}\right\|_{\mathcal{M}}<\left\|\left(h_{0}+\sigma\right)-\sigma\right\|_{\mathcal{M}}=\left\|h_{0}\right\|_{L^{1}} .
$$


Corollary 4 Given g, there exists a diffuse measure $\mu \geq 0$ such that $\varepsilon \mu$ is not good for any $\varepsilon>0$.

Proof. Using Remark 3, we can take sequences of disjoint compact sets $\left(K_{j}\right)$ in $\Omega$ and $L^{1}$-functions $\left(h_{j}\right)$, such that each pair $K_{j}, h_{j}$ satisfies the assumptions of Proposition 8 and

$$
\left\|h_{j}\right\|_{L^{1}} \leq \frac{1}{4^{j}} .
$$

Let $h=\sum_{j} h_{j} \in L^{1}(\Omega)$. For each $j \geq 1$, we fix a diffuse measure $\sigma_{j} \geq 0$ concentrated on $K_{j}$ such that $\left\|\sigma_{j}\right\|_{\mathcal{M}}=\frac{1}{2^{j}}$. Let $\mu=\sum_{j} \sigma_{j} \in \mathcal{M}(\Omega)$. Assume by contradiction that $\varepsilon \mu$ is good for some $\varepsilon>0$. Since

$$
\varepsilon \mu \geq \varepsilon \sigma_{j} \quad \forall j \geq 1,
$$

then $\varepsilon \sigma_{j}$ is also good. By Theorem 17, this gives

$$
\varepsilon<\frac{\left\|h_{j}\right\|_{L^{1}}}{\left\|\sigma_{j}\right\|_{\mathcal{M}}} \leq \frac{1}{2^{j}} \quad \forall j \geq 1
$$

As $j \rightarrow+\infty$, we get a contradiction.

Imposing some additional assumption on the nonlinearity $g$ one can construct a measure $\mu$ of the form $\mu=\theta \mathcal{H}^{\alpha}\left\lfloor_{K}\right.$, for some $\alpha>N-2$, such that $\mu \notin \mathcal{G}(g)$. To this purpose, one first needs a slight modification of Lemma 10:

Lemma 11 Assume $g$ is given by

$$
g(t)=\frac{1}{(1-t)^{\frac{2-\beta}{\beta}}}-1 \quad \forall t \in[0,1),
$$

where $\beta \in(0,2)$. Then, for any $\alpha \in(0, \beta)$, there exists $\tilde{v} \in C_{0}(\bar{\Omega})$ such that

$$
\Delta \tilde{v} \in L^{1}(\Omega), \quad \tilde{v} \leq 1 \text { in } \Omega, \quad \mathcal{H}^{N-2+\alpha}([\tilde{v}=1]) \in(0, \infty) \quad \text { and } \quad g(\tilde{v}) \in L^{1}(\Omega) .
$$

Proof. We just need to adapt the proof of Lemma 10. We shall consider both cases $N \geq 3$ and $N=2$ simultaneously. Let $\tilde{v}$ be given by (8.7). Using the same notation as before, we let

$$
\alpha_{j}=a_{m} 2^{-m N j}
$$

where

$$
\frac{\alpha}{N-2+\alpha}<m<\frac{(2-\alpha) \beta}{(2-\beta) \alpha} \frac{\alpha}{N-2+\alpha}
$$


and the constant $a_{m}$ is chosen so that (8.8) holds. Observe that the range of admissible $m$ given by (8.25) is nonempty since $0<\alpha<\beta<2$.

Next, we let $k_{j}=j$ and

$$
\ell_{k}=2^{-\frac{N k}{N-2+\alpha}} \quad \forall k \geq 1 .
$$

With $\left(\ell_{k}\right)$ defined as above, one can show that (see e.g. [18])

$$
\mathcal{H}^{N-2+\alpha}(F) \in(0, \infty)
$$

where $F=\bigcap_{j} F_{j}=[\tilde{v}=1]$. We now prove (8.11) (or, equivalently, (8.20) if $N=2)$. Note that, with our choices of $\left(\alpha_{j}\right)$ and $\left(\ell_{k}\right)$, the left-hand side of $(8.11)$ reduces to

$$
\sum_{j=1}^{\infty} 2^{m N j \frac{2-\beta}{\beta}} 2^{N j} 2^{-\frac{N^{2} j}{N-2+\alpha}}=\sum_{j=1}^{\infty} 2^{N j\left(m \frac{2-\beta}{\beta}-\frac{2-\alpha}{N-2+\alpha}\right)},
$$

which is finite, by (8.25). We now assume $N \geq 3$. Note that (8.10) becomes

$$
\sum_{j=1}^{\infty} 2^{-m N j} 2^{N j} 2^{-\frac{N(N-2) j}{N-2+\alpha}}=\sum_{j=1}^{\infty} 2^{-N j\left(m-\frac{\alpha}{N-2+\alpha}\right)}<\infty,
$$

which clearly holds in view of (8.25). Similarly, if $N=2$, then one easily checks that (8.19) is also satisfied. Proceeding as in the proof of Lemma 10, we conclude that (8.24) holds.

As a consequence, we have the following

Theorem 18 Given $\beta \in(0,2)$, let $g$ be given by (8.23). Then, for any $\alpha \in(0, \beta)$, there exist $\theta_{0}>0$ and $K \subset \Omega$ compact, $\mathcal{H}^{N-2+\alpha}(K) \in(0, \infty)$, such that

$$
\theta \mathcal{H}^{N-2+\alpha}\left\lfloor_{K} \in \mathcal{G}(g) \quad \text { implies } \theta<\theta_{0} .\right.
$$

Proof. Let

$$
\tilde{h}_{0}=[-\Delta \tilde{v}+g(\tilde{v})]^{+} \text {and } K=[\tilde{v}=1],
$$

where $\tilde{v}$ is given by Lemma 11 above. Proceeding as in the proof of Proposition 8 , we have

$$
\left(\tilde{h}_{0}+\theta \mathcal{H}^{N-2+\alpha}\left\lfloor_{K}\right)^{*}=\tilde{h}_{0} \quad \forall \theta>0 .\right.
$$

Therefore, if $\theta \mathcal{H}^{N-2+\alpha}\left\lfloor_{K}\right.$ is good, then as in the proof of Theorem 17 we conclude that

$$
\theta \mathcal{H}^{N-2+\alpha}(K)<\left\|\tilde{h}_{0}\right\|_{L^{1}} .
$$

In other words, (8.27) holds with $\theta_{0}=\frac{\left\|\tilde{h}_{0}\right\|_{L^{1}}}{\mathcal{H}^{N-2+\alpha}(K)}$. 


\section{Further properties of $\mu^{*}$ and $\mathcal{G}$}

In this section we prove some properties of the reduced measures, which should be compared with those in [4]. In particular, we start by showing that the reduced measure $\mu^{*}$ need not be the largest good measure $\leq \mu$, contrarily to what happens when $g$ is everywhere defined. In fact, we have

Proposition 9 There exists $\mu \in \mathcal{M}(\Omega), \mu \geq 0$, for which the set

$$
\{\lambda \in \mathcal{G}: \lambda \leq \mu\}
$$

has no largest element.

Proof. Let $K_{0}, h_{0}$ be given by Proposition 8. Let $\sigma$ be the capacitary measure associated to $K_{0}$. In particular, $\sigma$ is a nonnegative measure concentrated on $K_{0}$; moreover, $\sigma$ is good (see Proposition 7). Let $\mu=h_{0}+\sigma$. By Proposition 8, $\mu \notin \mathcal{G}(g)$. Assume by contradiction that the set given by (9.1) has a largest element, say $\nu \leq \mu$. Clearly, $\nu \geq h_{0}$ and $\nu \geq \sigma$. Thus,

$$
\nu \geq \sup \left\{h_{0}, \sigma\right\}=h_{0}+\sigma=\mu \text {. }
$$

We deduce that $\nu=\mu$, so that $\mu$ is a good measure. This is a contradiction.

Note that the same argument can be used to establish the next results (in what follows, $\sigma$ is the capacitary measure associated to $K_{0}$, with $K_{0}$ and $h_{0}$ being given by Proposition 8$)$.

Proposition 10 There exist good measures $\mu, \nu \geq 0$ such that $\sup \{\mu, \nu\}$ is not good.

Proof. Take $\mu=h_{0}, \nu=\sigma$ and use Proposition 8 .

Proposition 11 There exist diffuse measures $\mu, \nu \geq 0$ such that $\nu \leq \mu$ but $\mu^{*}-\nu^{*}$ is not $\geq 0$.

Proof. Take $\mu=h_{0}+\sigma$ and $\nu=\sigma$.

Similarly, the mapping $\mu \longmapsto \mu^{*}$ is not a contraction. More precisely, 
Proposition 12 There exist diffuse measures $\mu, \nu \geq 0$ such that

$$
\|\mu-\nu\|_{\mathcal{M}}<\left\|\mu^{*}-\nu^{*}\right\|_{\mathcal{M}}
$$

Proof. Take $\mu=h_{0}+\sigma$ and $\nu=\sigma$.

We conclude with the following

Proposition 13 The set $\mathcal{G}$ is not convex.

Proof. By Theorem 1, there exists $\mu$ diffuse such that $\mu \notin \mathcal{G}$. Applying Theorem 3 in [4], we can decompose $\mu$ as

$$
\mu=f+\Delta v,
$$

where $f \in L^{1}(\Omega), v \in H_{0}^{1}(\Omega) \cap C(\bar{\Omega})$ and $\|v\|_{L^{\infty}} \leq \frac{1}{3}$. In particular, $2 f \in \mathcal{G}$ and $\Delta(2 v) \in \mathcal{G}$; however,

$$
\frac{2 f+\Delta(2 v)}{2}=\mu \notin \mathcal{G}
$$

\section{Acknowledgments}

The second author (A.C.P.) was supported by the NSF grant DMS-0111298 and Sergio Serapioni, Honorary President of Società Trentina Lieviti-Trento (Italy). A.C.P. gratefully acknowledges the invitation and the warm hospitality of the Math Department at the University of Rome 1. The third author (A.P.) warmly thanks H. Brezis for the invitation at Rutgers University. Part of this work was carried out during these two occasions.

\section{References}

[1] A. Ancona, Une propriété d'invariance des ensembles absorbants par perturbation d'un opérateur elliptique. Comm. Partial Differential Equations 4 (1979), 321-337.

[2] L. Boccardo, On the regularizing effect of strongly increasing lower order terms. J. Evol. Equ. 3 (2003), 225-236.

[3] L. Boccardo, T. Gallouët, and L. Orsina, Existence and uniqueness of entropy solutions for nonlinear elliptic equations with measure data. Ann. Inst. H. Poincaré Anal. Non Linéaire 13 (1996), 539-551. 
[4] H. Brezis, M. Marcus, and A. C. Ponce, Nonlinear elliptic equations with measures revisited. To appear in Annals of Math. Studies, Princeton University Press. Part of the results were announced in a note by the same authors: $A$ new concept of reduced measure for nonlinear elliptic equations, C. R. Acad. Sci. Paris, Ser. I 339 (2004), 169-174.

[5] H. Brezis and A. C. Ponce, Remarks on the strong maximum principle. Differential Integral Equations 16 (2003), 1-12.

[6] H. Brezis and A. C. Ponce, Kato's inequality when $\Delta u$ is a measure. C. R. Math. Acad. Sci. Paris, Ser. I 338 (2004), 599-604.

[7] H. Brezis and W. A. Strauss, Semilinear second-order elliptic equations in $L^{1}$. J. Math. Soc. Japan 25 (1973), 565-590.

[8] L. Carleson, Selected problems on exceptional sets. Van Nostrand Mathematical Studies, No. 13, Van Nostrand, Princeton, 1967.

[9] G. Dal Maso, F. Murat, L. Orsina, and A. Prignet, Renormalized solutions of elliptic equations with general measure data. Ann. Scuola Norm. Sup. Pisa Cl. Sci. (4) 28 (1999), 741-808.

[10] P. Dall'Aglio and G. Dal Maso, Some properties of the solutions of obstacle problems with measure data. Ricerche Mat. 48 (1999), 99-116. Papers in memory of Ennio De Giorgi.

[11] P. Dall'Aglio and C. Leone, Obstacles problems with measure data and linear operators. Potential Anal. 17 (2002), 45-64.

[12] C. De La Vallée Poussin, Sur l'intégrale de Lebesgue. Trans. Amer. Math. Soc. 16 (1915), 435-501.

[13] C. Dellacherie and P.-A. Meyer, Probabilités et potentiel. Chapitres I à IV, Publications de l'Institut de Mathématique de l'Université de Strasbourg, No. XV, Actualités Scientifiques et Industrielles, No. 1372, Hermann, Paris, 1975.

[14] L. Dupaigne and A. C. Ponce, Singularities of positive supersolutions in elliptic PDEs. Selecta Math. (N.S.) 10 (2004), 341-358.

[15] L. C. Evans and R. F. Gariepy, Measure theory and fine properties of functions. Studies in Advanced Mathematics, CRC Press, Boca Raton, FL, 1992. 
[16] M. Fukushima, K. Sato, and S. Taniguchi, On the closable parts of preDirichlet forms and the fine supports of underlying measures. Osaka J. Math. 28 (1991), 517-535.

[17] M. Grun-Rehomme, Caractérisation du sous-différential d'intégrandes convexes dans les espaces de Sobolev. J. Math. Pures Appl. 56 (1977), 149-156.

[18] A. C. Ponce, How to construct good measures. In: Elliptic and Parabolic Equations (C. Bandle, H. Berestycki, B. Brighi, A. Brillard, M. Chipot, J.M. Coron, C. Sbordone, I. Shafrir, V. Valente, and G. Vergara-Caffarelli, eds.), Progress in Nonlinear Differential Equations and their Applications, 63, Birkhäuser, Basel, 2005, pp. 335-348. A special tribute to the work of Haïm Brezis.

[19] G. Stampacchia, Le problème de Dirichlet pour les équations elliptiques du second ordre à coefficients discontinus. Ann. Inst. Fourier (Grenoble) 15 (1965), $189-258$.

[20] J. L. Vázquez, On a semilinear equation in $\mathbb{R}^{2}$ involving bounded measures. Proc. Roy. Soc. Edinburgh Sect. A 95 (1983), 181-202.

L. Dupaigne

Laboratoire Amiénois de Mathématique Fondamentale et Appliquée

Université Picardie Jules Verne

Faculté de Mathématiques et d'Informatique

33, rue Saint-Leu 80039 Amiens Cedex 1

France

e-mail: louis.dupaigne@u-picardie.fr

A.C. Ponce

Institute for Advanced Study

Princeton, NJ 08540

USA

e-mail: augponce@math.ias.edu

A. Porretta

Dipartimento di Matematica

Università di Roma "Tor Vergata" 
Via della Ricerca Scientifica 1

00133 Roma

Italy

e-mail: porretta@mat.uniroma2.it 